\title{
Green's Relations on Regular Elements of Semigroup of Relational Hypersubstitutions for Algebraic Systems of Type $((m),(n))$
}

\author{
Jukkrit Daengsaen and Sorasak Leeratanavalee
}

\begin{abstract}
Any relational hypersubstitution for algebraic systems of type

$$
\left(\tau, \tau^{\prime}\right)=\left(\left(m_{i}\right)_{i \in I},\left(n_{j}\right)_{j \in J}\right)
$$

is a mapping which maps any $m_{i}$-ary operation symbol to an $m_{i}$-ary term and maps any $n_{j}$ ary relational symbol to an $n_{j}$-ary relational term preserving arities, where $I, J$ are indexed sets. Some algebraic properties of the monoid of all relational hypersubstitutions for algebraic systems of a special type, especially the characterization of its order and the set of all regular elements, were first studied by Phusanga and Koppitz[13] in 2018. In this paper, we study the Green's relations on the regular part of this monoid of a particular type $\left(\tau, \tau^{\prime}\right)=((m),(n))$, where $m, n \geq 2$.
\end{abstract}

\section{Introduction}

Identities are used to classify algebras into collections called varieties, hyperidentities are used to classify varieties of algebras into collections called hypervarieties. Hyperidentities have an interpretation in the theory of switching circuits and are also closely related to clone theory. The main tool which is used to study hyperidentities and hypervarieties is the concept of a hypersubstitution. The concept of hypersubstitutions of a given type $\tau$ in universal algebras was first introduced by Denecke et al.[5] in 1991. On the other hand, to classify algebraic systems into subclasses by logical sentences we can use the concept of hypersubstitutions for algebraic systems. The concept of hypersubstitutions for algebraic systems of a given type $\left(\tau, \tau^{\prime}\right)$ was first introduced by Denecke and Phusanga[6] in 2008. In 2018, Phusanga and Koppitz[13] introduced a new concept that generalize the notion of a hypersubstitution of type $\tau$ for universal algebras in a canonical way. Such hypersubstitution is called the relational hypersubstitution for algebraic systems of type $\left(\tau, \tau^{\prime}\right)$. The name was first used in [9]. The algebraic properties of the monoid of all relational hypersubstitutions for algebraic systems were studied intensively for several types. For examples,

2010 Mathematics Subject Classification. 20M05, 20M99, 20 N02.

Key words and phrases. Relational hypersubstitution, Algebraic system, Green's relations.

Corresponding author: Sorasak Leeratanavalee. 
all regular elements and the order of relational hypersubstitutions for algebraic systems of type $((2),(2))$ were determined by Phusanga and Koppitz in [13]. The order of linear relational hypersubstitutions for algebraic systems of type $((m),(n))$ was characterized by Lekkoksung and Phusanga in [9]. First of all, we recall the notion of an algebraic system of type $\left(\tau, \tau^{\prime}\right)$, which was first introduced by A.I. $\mathrm{Mal}^{\prime} \mathrm{cev}$ in 1951.

Definition 1. [10] Let $I, J$ be indexed sets. An algebraic system of type

$$
\left(\tau, \tau^{\prime}\right)=\left(\left(m_{i}\right)_{i \in I},\left(n_{j}\right)_{j \in J}\right)
$$

is a triple $\left(A,\left(f_{i}\right)_{i \in I},\left(\gamma_{j}\right)_{j \in J}\right)$ consisting of a nonempty set $A$, a sequence $\left(f_{i}\right)_{i \in I}$ of $m_{i}$-ary operations defined on $A$ and a sequence $\left(\gamma_{j}\right)_{j \in J}$ of $n_{j}$-ary relations on $A$. The pair $\left(\tau, \tau^{\prime}\right)$ is called the type of an algebraic system.

The concept of terms is one of the fundamental concepts of universal algebra. Terms may be consider as words formed by letters. To define terms we need variables and operation symbols. Let $X_{n}=\left\{x_{1}, \cdots, x_{n}\right\}$ be a finite set of variables for $n \in \mathbb{N}$, where $\mathbb{N}$ denotes the set of all positive integers, and let $X=\left\{x_{1}, x_{2}, \cdots\right\}$ be countably infinite. Let $\left\{f_{i}: i \in I\right\}$ be the set of $m_{i}$-ary operation symbols indexed by $I$. An $n$-ary term of type $\tau=\left(m_{i}\right)_{i \in I}$ is defined in the usual way by:

(i) Every $x_{k} \in X_{n}$ is an $n$-ary term of type $\tau$.

(ii) If $t_{1}, \cdots, t_{m_{i}}$ are $n$-ary terms of type $\tau$, then $f_{i}\left(t_{1}, \cdots, t_{m_{i}}\right)$ is an $n$-ary term of type $\tau$.

Let $W_{\tau}\left(X_{n}\right)$ be the set of all $n$-ary terms of type $\tau$ and let $W_{\tau}(X):=\bigcup_{n \geq 1} W_{\tau}\left(X_{n}\right)$ be the set of all terms of type $\tau$.

Definition 2. Let $I, J$ be indexed sets and $\left(\tau, \tau^{\prime}\right)=\left(\left(m_{i}\right)_{i \in I},\left(n_{j}\right)_{j \in J}\right)$ be a type, i.e. we have a sequence $\left(f_{i}\right)_{i \in I}$ of $m_{i}$-ary operation symbols and a sequence $\left(\gamma_{j}\right)_{j \in J}$ of $n_{j}$-ary relational symbols. If $t_{1}, \cdots, t_{n_{j}}$ are $n$-ary terms of type $\tau$, then we will call $\gamma_{j}\left(t_{1}, \cdots, t_{n_{j}}\right)$ is an $n$-ary relational term of type $\left(\tau, \tau^{\prime}\right)$.

Let $r F_{\left(\tau, \tau^{\prime}\right)}\left(X_{n}\right)$ be the set of all $n$-ary relational terms of type $\left(\tau, \tau^{\prime}\right)$. Let

$$
r F_{\left(\tau, \tau^{\prime}\right)}(X):=\bigcup_{n \in \mathbb{N}} r F_{\left(\tau, \tau^{\prime}\right)}\left(X_{n}\right)
$$

be the set of all relational terms of type $\left(\tau, \tau^{\prime}\right)$. A relational hypersubstitution for algebraic systems of type $\left(\tau, \tau^{\prime}\right)=\left(\left(m_{i}\right)_{i \in I},\left(n_{j}\right)_{j \in J}\right)$ is a mapping

$$
\sigma:\left\{f_{i}: i \in I\right\} \cup\left\{\gamma_{j}: j \in J\right\} \rightarrow W_{\tau}(X) \cup r F_{\left(\tau, \tau^{\prime}\right)}(X)
$$


with $\sigma\left(f_{i}\right) \in W_{\tau}\left(X_{m_{i}}\right)$ and $\sigma\left(\gamma_{j}\right) \in r F_{\left(\tau, \tau^{\prime}\right)}\left(X_{n_{j}}\right)$. Denote by $\operatorname{Relhyp}\left(\tau, \tau^{\prime}\right)$ the set of all relational hypersubstitutions for algebraic systems of type $\left(\tau, \tau^{\prime}\right)$. To defined a binary operation on $\operatorname{Relhyp}\left(\tau, \tau^{\prime}\right)$, we firstly recall the concept of superposition of terms and superposition of relational terms.

Let $m, n \in \mathbb{N}$. The superposition of terms $S_{n}^{m}: W_{\tau}\left(X_{m}\right) \times\left(W_{\tau}\left(X_{n}\right)\right)^{m} \rightarrow W_{\tau}\left(X_{n}\right)$ defined inductively by the following steps (see [11], pp.258):

(i) If $t=x_{k} \in X_{m}$ and $u_{1}, \cdots, u_{m} \in W_{\tau}\left(X_{n}\right)$, then $S_{n}^{m}\left(t, u_{1}, \cdots, u_{m}\right):=u_{k}$.

(ii) If $t=f_{i}\left(t_{1}, \cdots, t_{m_{i}}\right) \in W_{\tau}\left(X_{m}\right)$ and $u_{1}, \cdots, u_{m} \in W_{\tau}\left(X_{n}\right)$, then

$$
S_{n}^{m}\left(t, u_{1}, \cdots, u_{m}\right):=f_{i}\left(S_{n}^{m}\left(t_{1}, u_{1}, \cdots, u_{m}\right), \cdots, S_{n}^{m}\left(t_{m_{i}}, u_{1}, \cdots, u_{m}\right)\right) .
$$

Next, the superposition operation of relational terms

$$
R_{n}^{m}:\left(W_{\tau}\left(X_{m}\right) \cup r F_{\left(\tau, \tau^{\prime}\right)}\left(X_{m}\right)\right) \times\left(W_{\tau}\left(X_{n}\right)\right)^{m} \rightarrow W_{\tau}\left(X_{n}\right) \cup r F_{\left(\tau, \tau^{\prime}\right)}\left(X_{n}\right)
$$

defined by the following steps (see [11], pp.258 - 259):

(i) If $t \in W_{\tau}\left(X_{m}\right)$ and $u_{1}, \cdots, u_{m} \in W_{\tau}\left(X_{n}\right)$, then $R_{n}^{m}\left(t, u_{1}, \cdots, u_{m}\right):=S_{n}^{m}\left(t, u_{1}, \cdots, u_{m}\right)$.

(ii) If $\gamma_{j}\left(s_{1}, \cdots, s_{n_{j}}\right) \in r F_{\left(\tau, \tau^{\prime}\right)}\left(X_{m}\right)$ and $u_{1}, \cdots, u_{m} \in W_{\tau}\left(X_{n}\right)$, then

$$
R_{n}^{m}\left(\gamma_{j}\left(s_{1}, \cdots, s_{n_{j}}\right), u_{1}, \cdots, u_{m}\right):=\gamma_{j}\left(S_{n}^{m}\left(s_{1}, u_{1}, \cdots, u_{m}\right), \cdots, S_{n}^{m}\left(s_{n_{j}}, u_{1}, \cdots, u_{m}\right)\right) .
$$

For any $\sigma \in \operatorname{Relhyp}\left(\tau, \tau^{\prime}\right)$, we define a mapping

$$
\widehat{\sigma}: W_{\tau}(X) \cup r F_{\left(\tau, \tau^{\prime}\right)}(X) \rightarrow W_{\tau}(X) \cup r F_{\left(\tau, \tau^{\prime}\right)}(X)
$$

inductively defined as follows:

(i) $\widehat{\sigma}\left[x_{k}\right]:=x_{k}$, for $k \in \mathbb{N}$,

(ii) $\widehat{\sigma}\left[f_{i}\left(t_{1}, \cdots, t_{m_{i}}\right)\right]:=R_{m}^{m_{i}}\left(\sigma\left(f_{i}\right), \widehat{\sigma}\left[t_{1}\right], \cdots, \widehat{\sigma}\left[t_{m_{i}}\right]\right)=S_{m}^{m_{i}}\left(\sigma\left(f_{i}\right), \widehat{\sigma}\left[t_{1}\right], \cdots, \widehat{\sigma}\left[t_{m_{i}}\right]\right)$,

(iii) $\widehat{\sigma}\left[\gamma_{j}\left(s_{1}, \cdots, s_{n_{j}}\right)\right]:=R_{n}^{n_{j}}\left(\sigma\left(\gamma_{j}\right), \widehat{\sigma}\left[s_{1}\right], \cdots, \widehat{\sigma}\left[s_{n_{j}}\right]\right)$.

A binary operation $\circ_{h}$ on $\operatorname{Relhyp}\left(\tau, \tau^{\prime}\right)$ is defined by $\sigma \circ_{h} \rho:=\widehat{\sigma} \circ \rho$ where $\circ$ denotes the usual composition of mappings and $\sigma, \rho \in \operatorname{Relhyp}\left(\tau, \tau^{\prime}\right)$. Let $\sigma_{i d}$ be the relational hypersubstitution which maps each $m_{i}$-ary operation symbol $f_{i}$ to the term $f_{i}\left(x_{1}, \cdots, x_{m_{i}}\right)$ and maps each $n_{j}$-ary relation symbol $\gamma_{j}$ to the relational term $\gamma_{j}\left(x_{1}, \cdots, x_{n_{j}}\right)$. Then the structure $\operatorname{Relhyp}\left(\tau, \tau^{\prime}\right)=$ $\left(\operatorname{Relhyp}\left(\tau, \tau^{\prime}\right), \circ_{h}, \sigma_{i d}\right)$ forms a monoid. In [3], the authors studied the regularity of relational hypersubstitution for algebraic systems of type $((m),(n))$. In this paper, we study the Green's relations on the set of all regular relational hypersubstitutions for algebraic systems of type $((m),(n))$, for arbitrary natural numbers $m, n \geq 2$. 


\section{Preliminaries and Notations}

Green's relations of a semigroup $S$ are equivalence relations on the set $S$ which were first defined by Green. According to such definitions, the $\mathcal{L}$-relation was defined as follows. Let $(S, \cdot)$ be a monoid. For any $a, b \in S$,

$$
a \mathcal{L} b \text { if and only if } S \cdot a=S \cdot b,
$$

or equivalently, $a \mathcal{L} b$ if and only if $a=x \cdot b$ and $b=y \cdot a$ for some $x, y \in S$. Furthermore, the $\mathcal{R}$-relation was defined as follows.

$$
a \mathcal{R} b \text { if and only if } a \cdot S=b \cdot S
$$

or equivalently, $a \mathcal{R} b$ if and only if $a=b \cdot x$ and $b=a \cdot y$ for some $x, y \in S$. Moreover, the $\mathcal{J}$-relation was defined as follows.

$$
a \mathcal{J} b \text { if and only if } S \cdot a \cdot S=S \cdot b \cdot S
$$

or equivalently, $a \mathcal{J} b$ if and only if $a=x \cdot b \cdot y$ and $b=u \cdot a \cdot v$ for some $x, y, u, v \in S$. Finally, letting $\mathcal{H}=\mathcal{L} \cap \mathcal{R}$ and $\mathcal{D}=\mathcal{L} \circ \mathcal{R}$, where $\circ$ is the composition of relations. Since the relations $\mathcal{L}$ and $\mathcal{R}$ commute, it follows that $\mathcal{L} \circ \mathcal{R}=\mathcal{R} \circ \mathcal{L}$.

Remark 1. Let $S$ be a semigroup. Then $\mathcal{H} \subseteq \mathcal{L} \subseteq \mathcal{D} \subseteq \mathcal{J}$ and $\mathcal{H} \subseteq \mathcal{R} \subseteq \mathcal{D} \subseteq \mathcal{J}$.

Throughout this paper, we focus to the algebraic systems of type $((m),(n))$. Let $f$ be an $m$-ary operation symbol and let $\gamma$ be an $n$-ary relational symbol. For any $t \in W_{(m)}\left(X_{m}\right)$ and $F \in r F_{((m),(n))}\left(X_{n}\right)$, we introduce the following notation:

(i) $\sigma_{t}:=$ the hypersubstitution of type $(m)$ which maps $f$ to the term $t \in W_{(m)}\left(X_{m}\right)$,

(ii) $\sigma_{t, F}:=$ the relational hypersubstitution for algebraic systems of type $((m),(n))$ which maps $f$ to the term $t \in W_{(m)}\left(X_{m}\right)$ and maps $\gamma$ to the relational term $F \in r F_{((m),(n))}\left(X_{n}\right)$,

(iii) $I(t):=$ the set of all indices of variables occurring in a term $t$,

(iv) $I(F):=$ the set of all indices of variables occurring in a relational term $F$,

(v) $\pi(t):=$ the term such that each $x_{k}, k \in I(t)$, is replaced by $x_{\pi(k)}$ where $\pi$ is an injective map from $I(t)$ into $\{1, \cdots, m\}$, i.e. $\pi(t)=S_{m}^{m}\left(t, y_{1}, \cdots, y_{m}\right)$ with $y_{1}, \cdots, y_{m} \in X_{m}$ and $y_{i}:=x_{\pi(i)}$ for $i \in I(t)$.

(vi) $\phi(F):=$ the relational term such that each $x_{k}, k \in I(F)$, is replaced by $x_{\phi(k)}$ where $\phi$ is a injective map from $I(F)$ into $\{1, \cdots, n\}$, i.e. $\phi(F)=R_{m}^{m}\left(F, y_{1}, \cdots, y_{m}\right)$ with $y_{1}, \cdots, y_{m} \in X_{m}$ and $y_{i}:=x_{\phi(i)}$ for $i \in I(F)$. 
In 2015, W. Wongpinit and S. Leeratanavalee [16] introduced the concept of the $i-$ most of terms.

Definition 3. Let $t \in W_{(m)}\left(X_{m}\right)$ and $1 \leq i \leq m$. Then $i-\operatorname{most}(t)$ is defined inductively by:

(i) If $t$ is a variable, then $i-\operatorname{most}(t)=t$.

(ii) If $t=f\left(t_{1}, \cdots, t_{m}\right)$, then $i-\operatorname{most}(t)=i-\operatorname{most}\left(t_{i}\right)$.

Example 1. Let $\tau=(3)$ be a type with a ternary operation symbol $f$.

Let $t=f\left(x_{2}, f\left(x_{3}, f\left(x_{2}, x_{3}, x_{1}\right), x_{3}\right), f\left(x_{3}, x_{1}, x_{1}\right)\right) \in W_{(3)}\left(X_{3}\right)$. Then

$1-\operatorname{most}(t)=1-\operatorname{most}\left(x_{2}\right)=x_{2}$,

$2-\operatorname{most}(t)=2-\operatorname{most}\left(f\left(x_{3}, f\left(x_{2}, x_{3}, x_{1}\right), x_{3}\right)\right)=2-\operatorname{most}\left(f\left(x_{2}, x_{3}, x_{1}\right)\right)=x_{3}$ and

$3-\operatorname{most}(t)=3-\operatorname{most}\left(f\left(x_{3}, x_{1}, x_{1}\right)\right)=x_{1}$.

Furthermore, we have $\widehat{\sigma}_{x_{1}}[t]=\widehat{\sigma}_{x_{1}}\left[f\left(x_{2}, f\left(x_{3}, f\left(x_{2}, x_{3}, x_{1}\right), x_{3}\right), f\left(x_{3}, x_{1}, x_{1}\right)\right)\right]=$

$S_{3}^{3}\left(\sigma_{x_{1}}(f), \widehat{\sigma}_{x_{1}}\left[x_{2}\right], \widehat{\sigma}_{x_{1}}\left[f\left(x_{3}, f\left(x_{2}, x_{3}, x_{1}\right), x_{3}\right)\right], \widehat{\sigma}_{x_{1}}\left[f\left(x_{3}, x_{1}, x_{1}\right)\right]\right)=$

$S_{3}^{3}\left(x_{1}, x_{2}, \widehat{\sigma}_{x_{1}}\left[f\left(x_{3}, f\left(x_{2}, x_{3}, x_{1}\right), x_{3}\right)\right], \widehat{\sigma}_{x_{1}}\left[f\left(x_{3}, x_{1}, x_{1}\right)\right]\right)=x_{2}=1-\operatorname{most}(t)$.

Similarly, we have $\widehat{\sigma}_{x_{2}}[t]=x_{3}=2-\operatorname{most}(t)$ and $\widehat{\sigma}_{x_{3}}[t]=x_{1}=3-\operatorname{most}(t)$.

Remark 2. By Example 1, we have $\widehat{\sigma}_{x_{i}}[t]=i-\operatorname{most}(t)$. Furthermore, we have $\widehat{\sigma}_{x_{i}, F}[t]=$ $i-\operatorname{most}(t)$, where $x_{i}, t \in W_{(m)}\left(X_{m}\right)$ and $F \in r F_{((m),(n))}\left(X_{m}\right)$.

Lemma 2.1. [3] Let $\sigma_{t, F} \in \operatorname{Relhyp}((m),(n))$ where $t \in W_{(m)}\left(X_{m}\right)$ and $F \in r F_{((m),(n))}\left(X_{n}\right)$. Let $s \in W_{(m)}\left(X_{m}\right)$. If $i-\operatorname{most}(t)=x_{j}$ then $j-\operatorname{most}(s)=i-\operatorname{most}\left(\widehat{\sigma}_{t, F}[s]\right)$.

Remark 3. Let $\sigma_{s}$ and $\sigma_{t}$ be hypersubstitutions of type $\tau=(n)$. Then $I\left(\left(\sigma_{s} \circ_{h} \sigma_{t}\right)(f)\right) \subseteq I(t)$.

By applying the above remark, we obtain the following result.

Lemma 2.2. Let $\sigma_{t, F}, \sigma_{w, H} \in \operatorname{Relhyp}((m),(n))$. Then $I\left(\left(\sigma_{t, F} \circ_{h} \sigma_{w, H}\right)(f)\right) \subseteq I\left(\sigma_{w, H}(f)\right)$ and $I\left(\left(\sigma_{t, F} \circ_{h} \sigma_{w, H}\right)(\gamma)\right) \subseteq I\left(\sigma_{w, H}(\gamma)\right)$.

Proof. It is obvious if $w, t \in X_{m}$. Let $t=f\left(t_{1}, \cdots, t_{m}\right), w=f\left(w_{1}, \cdots, w_{m}\right) \in W_{(m)}\left(X_{m}\right) \backslash$ $X_{m}$ and $F=\gamma\left(s_{1}, \cdots, s_{n}\right), H=\gamma\left(h_{1}, \cdots, h_{n}\right) \in r F_{((m),(n))}\left(X_{n}\right)$ where $s_{1}, \cdots, s_{n}, h_{1}, \cdots, h_{n}$ $\in W_{(m)}\left(X_{n}\right)$. First, we consider

$$
\begin{aligned}
\left(\sigma_{t, F} \circ_{h} \sigma_{w, H}\right)(f) & =\widehat{\sigma}_{t, F}\left[\sigma_{w, H}(f)\right] \\
& =\widehat{\sigma}_{t, F}\left[f\left(w_{1}, \cdots, w_{m}\right)\right] \\
& =S_{m}^{m}\left(t, \widehat{\sigma}_{t, F}\left[w_{1}\right], \cdots, \widehat{\sigma}_{t, F}\left[w_{m}\right]\right) .
\end{aligned}
$$

Clearly, $\widehat{\sigma}_{t, F}\left[w_{k}\right]=\widehat{\sigma}_{t}\left[w_{k}\right]$ for all $k=1, \cdots, m$. By Remark 3 , we have $I\left(\widehat{\sigma}_{t, F}\left[w_{k}\right]\right)=I\left(\widehat{\sigma}_{t}\left[w_{k}\right]\right)=$ $I\left(\left(\sigma_{t} \circ_{h} \sigma_{w_{k}}\right)(f)\right) \subseteq I\left(w_{k}\right)$. Hence $I\left(\left(\sigma_{t, F} \circ_{h} \sigma_{w, H}\right)(f)\right)=$ 
$I\left(S_{m}^{m}\left(t, \widehat{\sigma}_{t, F}\left[w_{1}\right], \cdots, \widehat{\sigma}_{t, F}\left[w_{m}\right]\right)\right) \subseteq \bigcup_{k=1}^{m} I\left(w_{k}\right)=I(w)=I\left(\sigma_{w, H}(f)\right)$. On the other hand, we consider

$$
\begin{aligned}
\left(\sigma_{t, F} \circ_{h} \sigma_{w, H}\right)(\gamma) & =\widehat{\sigma}_{t, F}\left[\sigma_{w, H}(\gamma)\right] \\
& =\widehat{\sigma}_{t, F}\left[\gamma\left(h_{1}, \cdots, h_{n}\right)\right] \\
& =R_{n}^{n}\left(F, \widehat{\sigma}_{t, F}\left[h_{1}\right], \cdots, \widehat{\sigma}_{t, F}\left[h_{n}\right]\right) \\
& =\gamma\left(S_{n}^{m}\left(s_{1}, \widehat{\sigma}_{t, F}\left[h_{1}\right], \cdots, \widehat{\sigma}_{t, F}\left[h_{n}\right]\right), \cdots, S_{n}^{m}\left(s_{n}, \widehat{\sigma}_{t, F}\left[h_{1}\right], \cdots, \widehat{\sigma}_{t, F}\left[h_{n}\right]\right)\right) .
\end{aligned}
$$

By the same process, we have $I\left(S_{n}^{m}\left(s_{k}, \widehat{\sigma}_{t, F}\left[h_{1}\right], \cdots, \widehat{\sigma}_{t, F}\left[h_{n}\right]\right)\right) \subseteq \bigcup_{k=1}^{n} I\left(h_{k}\right)=I(H)$. Thus $I\left(\left(\sigma_{t, F} \circ_{h} \sigma_{w, H}\right)(\gamma)\right) \subseteq I(H)=I\left(\sigma_{w, H}(\gamma)\right)$ and the proof is complete.

\section{Green's relations on $\operatorname{Reg}(\operatorname{Relhyp}((m),(n)))$}

In this section, we study Green's relations on regular elements of semigroup of all relational hypersubstitutions for algebraic systems of type $((m),(n))$.

Let $\sigma_{t, F} \in \operatorname{Relhyp}((m),(n))$, we denote

$R_{X}:=\left\{\sigma_{t, F} \mid t=x_{i}\right.$ and $F=\gamma\left(s_{1}, \cdots, s_{n}\right)$ with $I(F)=\left\{b_{1}, \cdots, b_{l}\right\}$ such that $j-$ $\operatorname{most}\left(s_{b^{\prime}{ }_{k}}\right)=x_{b_{k}}$ for all $k=1, \cdots, l$ and for some distinct $b^{\prime}{ }_{1}, \cdots, b_{l}^{\prime} \in\{1, \cdots, n\}$ where $j \in\{1, \cdots, m\}\}$,

$R_{T}:=\left\{\sigma_{t, F} \mid t=f\left(t_{1}, \cdots, t_{m}\right)\right.$ and $F=\gamma\left(s_{1}, \cdots, s_{n}\right)$ with $I(t)=\left\{a_{1}, \cdots, a_{k}\right\}$ and $I(F)=\left\{b_{1}, \cdots, b_{l}\right\}$ such that $t_{a^{\prime}{ }_{i}}=x_{a_{i}}$ and $s_{b^{\prime}{ }_{j}}=x_{b_{j}}$ for all $i=1, \cdots, k, j=1, \cdots, l$, for some distinct $a^{\prime}{ }_{1}, \cdots, a^{\prime}{ }_{k} \in\{1, \cdots, m\}$ and some distinct $\left.b^{\prime}{ }_{1}, \cdots, b_{l}^{\prime} \in\{1, \cdots, n\}\right\}$.

In [3], the authors showed that $\operatorname{Reg}(\operatorname{Re} \operatorname{lhyp}((m),(n))):=R_{X} \cup R_{T}$ is the set of all regular elements in $\operatorname{Relhyp}((m),(n))$, but the set $\operatorname{Reg}(\operatorname{Relhyp}((m),(n)))$ is not a subsemigroup of $\operatorname{Relhyp}((m),(n))$. We can illustrate it by the following example.

Example 2. Let $\left(\tau, \tau^{\prime}\right)=((2),(2))$ be a type with a binary operation symbol $f$ and a binary relational symbol $\gamma$. Let $\sigma_{t, F}, \sigma_{u, H} \in \operatorname{Reg}(\operatorname{Relhyp}((2),(2)))$ such that $t=f\left(x_{1}, f\left(x_{1}, x_{1}\right)\right)$, $u=f\left(f\left(x_{2}, x_{2}\right), x_{2}\right)$ and $F=\gamma\left(x_{1}, f\left(x_{1}, x_{1}\right)\right), H=\gamma\left(f\left(x_{2}, x_{2}\right), x_{2}\right)$. Then

$$
\begin{aligned}
\left(\sigma_{t, F} \circ_{h} \sigma_{u, H}\right)(f) & =\widehat{\sigma}_{t, F}\left[f\left(f\left(x_{2}, x_{2}\right), x_{2}\right)\right] \\
& =S_{2}^{2}\left(f\left(x_{1}, f\left(x_{1}, x_{1}\right)\right), S_{2}^{2}\left(f\left(x_{1}, f\left(x_{1}, x_{1}\right)\right), x_{2}, x_{2}\right), x_{2}\right) \\
& =S_{2}^{2}\left(f\left(x_{1}, f\left(x_{1}, x_{1}\right)\right), f\left(x_{2}, f\left(x_{2}, x_{2}\right)\right), x_{2}\right) \\
& =f\left(f\left(x_{2}, f\left(x_{2}, x_{2}\right)\right), f\left(f\left(x_{2}, f\left(x_{2}, x_{2}\right)\right), f\left(x_{2}, f\left(x_{2}, x_{2}\right)\right)\right)\right)
\end{aligned}
$$


and

$$
\begin{aligned}
\left(\sigma_{t, F} \circ_{h} \sigma_{u, H}\right)(\gamma) & =\widehat{\sigma}_{t, F}\left[\gamma\left(f\left(x_{2}, x_{2}\right), x_{2}\right)\right] \\
& =R_{2}^{2}\left(\gamma\left(x_{1}, f\left(x_{1}, x_{1}\right)\right), S_{2}^{2}\left(f\left(x_{1}, f\left(x_{1}, x_{1}\right)\right), x_{2}, x_{2}\right), x_{2}\right) \\
& =R_{2}^{2}\left(\gamma\left(x_{1}, f\left(x_{1}, x_{1}\right)\right), f\left(x_{2}, f\left(x_{2}, x_{2}\right)\right), x_{2}\right) \\
& =\gamma\left(f\left(x_{2}, f\left(x_{2}, x_{2}\right)\right), f\left(f\left(x_{2}, f\left(x_{2}, x_{2}\right)\right), f\left(x_{2}, f\left(x_{2}, x_{2}\right)\right)\right)\right) .
\end{aligned}
$$

So $\sigma_{t, F} \circ_{h} \sigma_{u, H} \notin \operatorname{Reg}(\operatorname{Relhyp}((2),(2)))$. Thus $\operatorname{Reg}(\operatorname{Relhyp}((2),(2)))$ is not a subsemigroup of $\operatorname{Relhyp}((2),(2))$.

We begin with characterizing the Green's relations when two regular relational hypersubstitutions are elements of $R_{X}$ as follows.

Theorem 3.1. Let $\sigma_{x_{i}, F}, \sigma_{x_{j}, H} \in R_{X}$. Then $\sigma_{x_{i}, F} \mathcal{L} \sigma_{x_{j}, H}$ if and only if $i=j$ and $I(F)=I(H)$.

Proof. Let $\sigma_{x_{i}, F} \mathcal{L} \sigma_{x_{j}, H}$. Then there exist $\sigma_{u, G}, \sigma_{v, O} \in \operatorname{Relhyp}((m),(n))$ such that $\sigma_{x_{i}, F}=$ $\sigma_{u, G} \circ_{h} \sigma_{x_{j}, H}$ and $\sigma_{x_{j}, H}=\sigma_{v, O} \circ_{h} \sigma_{x_{i}, F}$. We have $x_{i}=\sigma_{x_{i}, F}(f)=\left(\sigma_{u, G} \circ_{h} \sigma_{x_{j}, H}\right)(f)=$ $\widehat{\sigma}_{u, G}\left[\sigma_{x_{j}, H}(f)\right]=x_{j}$. So $i=j$. Next, we consider $F=\sigma_{x_{i}, F}(\gamma)=\left(\sigma_{u, G} \circ_{h} \sigma_{x_{j}, H}\right)(\gamma)$ and $H=\sigma_{x_{j}, H}(\gamma)=\left(\sigma_{v, O} \circ_{h} \sigma_{x_{i}, F}\right)(\gamma)$. By Lemma 2.2, we have $I(F) \subseteq I(H)$ and $I(H) \subseteq I(F)$. So $I(H)=I(F)$.

Conversely, let $\sigma_{x_{i}, F}, \sigma_{x_{j}, H} \in R_{X}$ with $i=j, F=\gamma\left(s_{1}, \cdots, s_{n}\right)$ and $H=\gamma\left(h_{1}, \cdots, h_{n}\right)$ where $I(F)=\left\{b_{1}, \cdots, b_{l}\right\}=I(H)$. Since $\sigma_{x_{i}, F}, \sigma_{x_{j}, H} \in R_{x}$, there exist $p, q \in\{1, \cdots, m\}$ and the set of all distinct elements $\left\{b^{\prime}{ }_{1}, \cdots, b_{l}^{\prime}\right\},\left\{b^{\prime \prime}{ }_{1}, \cdots, b_{l}^{\prime \prime}\right\} \subseteq\{1, \cdots, n\}$ such that $p-$ $\operatorname{most}\left(s_{b_{k}^{\prime}}\right)=x_{b_{k}}$ and $q-\operatorname{most}\left(h_{b_{k}^{\prime \prime}}\right)=x_{b_{k}}$ for all $k=1, \cdots, l$. We will show that $\sigma_{x_{i}, F} \mathcal{L} \sigma_{x_{j}, H}$. First, we define $\pi:\left\{b_{1}, \cdots, b_{l}\right\} \rightarrow\left\{b_{1}^{\prime}, \cdots, b_{l}^{\prime}\right\}$ by $\pi\left(b_{k}\right)=b_{k}^{\prime}$, for all $k=1, \cdots, l$, and define $\phi:\left\{b_{1}, \cdots, b_{l}\right\} \rightarrow\left\{b_{1}^{\prime \prime}, \cdots, b_{l}^{\prime \prime}\right\}$ by $\phi\left(b_{k}\right)=b_{k}^{\prime \prime}$ for all $k=1, \cdots, l$. Then $\pi$ and $\phi$ are bijective and $p-\operatorname{most}\left(s_{b_{k}^{\prime}}\right)=x_{b_{k}}=x_{\pi^{-1}\left(b_{k}^{\prime}\right)}$ and $q-\operatorname{most}\left(h_{b_{k}^{\prime \prime}}\right)=x_{b_{k}}=x_{\phi^{-1}\left(b_{k}^{\prime \prime}\right)}$ for all $k=1, \cdots, l$. Choose $\sigma_{x_{q}, \phi(F)}, \sigma_{x_{p}, \pi(H)} \in \operatorname{Relhyp}((m),(n))$. Then

$$
\begin{aligned}
\left(\sigma_{x_{q}, \phi(F)} \circ_{h} \sigma_{x_{j}, H}\right)(\gamma)= & \widehat{\sigma}_{x_{q}, \phi(F)}\left[\sigma_{x_{j}, H}(\gamma)\right] \\
= & \widehat{\sigma}_{x_{q}, \phi(F)}[H] \\
= & R_{n}^{n}\left(\phi(F), q-\operatorname{most}\left(h_{1}\right), \cdots, q-\operatorname{most}\left(h_{n}\right)\right) \\
= & \phi^{-1}(\phi(F)) \\
& \operatorname{since} q-\operatorname{most}\left(h_{b_{k}^{\prime \prime}}\right)=x_{\phi^{-1}\left(b_{k}\right)} \text { for all } k=1, \cdots, l \\
& \text { and } I(\phi(F))=\left\{b_{1}^{\prime \prime}, \cdots, b_{l}^{\prime \prime}\right\} \\
= & F=\left(\sigma_{x_{i}, F}\right)(\gamma) .
\end{aligned}
$$

It is obvious that $\left(\sigma_{x_{q}, \phi(F)}{ }^{\circ}{ }_{h} \sigma_{x_{j}, H}\right)(f)=x_{j}=x_{i}=\left(\sigma_{x_{i}, F}\right)(f)$. So $\sigma_{x_{q}, \phi(F)}{ }^{{ }_{h}} \sigma_{x_{j}, H}=\sigma_{x_{i}, F}$. Similarly, $\sigma_{x_{p}, \pi(H)}{ }^{\circ}{ }_{h} \sigma_{x_{i}, F}=\sigma_{x_{j}, H}$. Therefore $\sigma_{x_{i}, F} \mathcal{L} \sigma_{x_{j}, H}$. 
Theorem 3.2. Let $\sigma_{x_{i}, F}, \sigma_{x_{j}, H} \in R_{X}$. Then $\sigma_{x_{i}, F} \mathcal{R} \sigma_{x_{j}, H}$ if and only if

(i) $|I(H)|=|I(F)|$.

(ii) $H=\pi(F)$ where $\pi$ is a bijective map from $I(F)$ to $I(H)$.

Proof. Let $\sigma_{x_{i}, F} \mathcal{R} \sigma_{x_{j}, H}$ where $F=\gamma\left(s_{1}, \cdots, s_{n}\right), H=\gamma\left(h_{1}, \cdots, h_{n}\right) \in r F_{((m),(n))}\left(X_{n}\right)$. Then there exist $\sigma_{u, G}, \sigma_{v, O} \in \operatorname{Re} \operatorname{lhyp}((m),(n))$ such that $\sigma_{x_{i}, F}=\sigma_{x_{j}, H} \circ_{h} \sigma_{u, G}$ and $\sigma_{x_{j}, H}=$ $\sigma_{x_{i}, F^{\circ}} \sigma_{v, O}$ where $u, v \in W_{(m)}\left(X_{m}\right)$ and $G=\gamma\left(g_{1}, \cdots, g_{n}\right), O=\gamma\left(o_{1}, \cdots, o_{n}\right) \in r F_{((m),(n))}\left(X_{n}\right)$. Then

$$
\begin{aligned}
F & =\sigma_{x_{i}, F}(\gamma) \\
& =\left(\sigma_{x_{j}, H} \circ_{h} \sigma_{u, G}\right)(\gamma) \\
& =\widehat{\sigma}_{x_{j}, H}\left[\sigma_{u, G}(\gamma)\right] \\
& =\widehat{\sigma}_{x_{j}, H}\left[\gamma\left(g_{1}, \cdots, g_{n}\right)\right] \\
& =R_{n}^{n}\left(H, \widehat{\sigma}_{x_{j}, H}\left[g_{1}\right], \cdots, \widehat{\sigma}_{x_{j}, H}\left[g_{1}\right]\right) \\
& =R_{n}^{n}\left(H, j-\operatorname{most}\left(g_{1}\right), \cdots, j-\operatorname{most}\left(g_{n}\right)\right),
\end{aligned}
$$

where $s_{p}=S_{n}^{n}\left(h_{p}, j-\operatorname{most}\left(g_{1}\right), \cdots, j-\operatorname{most}\left(g_{n}\right)\right)$, for all $p=1, \cdots, n$, and

$$
\begin{aligned}
H & =\sigma_{x_{j}, H}(\gamma) \\
& =\left(\sigma_{x_{i}, F} \circ_{h} \sigma_{v, O}\right)(\gamma) \\
& =\widehat{\sigma}_{x_{i}, F}\left[\sigma_{v, O}(\gamma)\right] \\
& =\widehat{\sigma}_{x_{i}, F}\left[\gamma\left(o_{1}, \cdots, o_{n}\right)\right] \\
& =R_{n}^{n}\left(F, \widehat{\sigma}_{x_{i}, F}\left[o_{1}\right], \cdots, \widehat{\sigma}_{x_{i}, F}\left[o_{1}\right]\right) \\
& =R_{n}^{n}\left(F, i-\operatorname{most}\left(o_{1}\right), \cdots, i-\operatorname{most}\left(o_{n}\right)\right)
\end{aligned}
$$

where $h_{p}=S_{n}^{n}\left(s_{p}, i-\operatorname{most}\left(o_{1}\right), \cdots, i-\operatorname{most}\left(o_{n}\right)\right)$ for all $p=1, \cdots, n$.

Since $\sigma_{x_{i}, F} \in R_{x}$, there exists $k \in\{1, \cdots, m\}$ and some distinct $b_{1}^{\prime}, \cdots, b_{l}^{\prime} \in\{1, \cdots, n\}$ such that $k-\operatorname{most}\left(s_{b_{q}^{\prime}}\right)=x_{b_{q}}$, for all $q=1, \cdots, l$, where $I(F)=\left\{b_{1}, \cdots, b_{l}\right\}$. Then

$$
x_{b_{q}}=k-\operatorname{most}\left(s_{b_{q}^{\prime}}\right)=S_{n}^{n}\left(k-\operatorname{most}\left(h_{b_{q}^{\prime}}\right), j-\operatorname{most}\left(g_{1}\right), \cdots, j-\operatorname{most}\left(g_{n}\right)\right) .
$$

Hence for each $q=1, \cdots, l$, there exists $d_{q} \in I(H)$ such that $k-\operatorname{most}\left(h_{b_{q}^{\prime}}\right)=x_{d_{q}}$ then $x_{b_{q}}=$ $j-\operatorname{most}\left(g_{d_{q}}\right)$. If there exist $d_{q}=d_{r}$ for some $q \neq r \in\{1, \cdots, l\}$ such that $k-\operatorname{most}\left(h_{b_{q}^{\prime}}\right)=$ $x_{d_{q}}=x_{d_{r}}=k-\operatorname{most}\left(h_{b_{r}^{\prime}}\right)$ then $x_{b_{q}}=j-\operatorname{most}\left(g_{d_{q}}\right)=j-\operatorname{most}\left(g_{d_{r}}\right)=x_{b_{r}}$. This is a contradiction with $b_{q} \neq b_{r} \in I(F)$ for all $q \neq r \in\{1, \cdots, l\}$. Hence we obtain the set of all distinct elements $\left\{d_{1}, \cdots, d_{l}\right\}$ of $I(H)$. It implies that $|I(F)|=\left|\left\{d_{1}, \cdots, d_{l}\right\}\right| \leq|I(H)|$. Similarly, we can show that $|I(H)| \leq|I(F)|$. So $|I(H)|=|I(F)|$ and the proof of (i) is complete. Next, we 
will prove (ii). Without loss of generality, we assume that $I(H)=\left\{d_{1}, \cdots, d_{l}\right\}$. Define a bijective $\operatorname{map} \phi: I(H) \rightarrow I(F)$ by $\phi\left(d_{q}\right)=b_{q}$ for all $q=1, \cdots, l$. Then $j-\operatorname{most}\left(g_{d_{q}}\right)=x_{b_{q}}=x_{\phi\left(d_{q}\right)}$ for all $q=1, \cdots, l$. Since $I(H)=\left\{d_{1}, \cdots, d_{l}\right\}$, we have $F=R_{n}^{n}\left(H, j-\operatorname{most}\left(g_{1}\right), \cdots, j-\right.$ $\left.\operatorname{most}\left(g_{n}\right)\right)=\phi(H)$. It follows that $H=\pi(F)$ where $\pi=\phi^{-1}$ is a bijective map from $I(F)$ to $I(H)$.

Conversely, assume that the conditions hold. Let $\sigma_{x_{i}, F}, \sigma_{x_{j}, H} \in R_{X}$ with $H=\pi(F)$ where $\pi$ is a bijective map from $I(F)$ to $I(H), F=\gamma\left(s_{1}, \cdots, s_{n}\right) \in r F_{((m),(n))}\left(X_{n}\right), I(F)=$ $\left\{b_{1}, \cdots, b_{l}\right\}$ and $I(H)=\left\{d_{1}, \cdots, d_{l}\right\}$.

Choose $\sigma_{x_{i}, P}, \sigma_{x_{j}, Q} \in \operatorname{Relhyp}((m),(n))$ where $P=\gamma\left(p_{1}, \cdots, p_{n}\right), Q=\gamma\left(q_{1}, \cdots, q_{n}\right) \in$ $r F_{((m),(n))}\left(X_{n}\right)$ with $j-\operatorname{most}\left(p_{d_{k}}\right)=x_{\pi^{-1}\left(d_{k}\right)}$ and $i-\operatorname{most}\left(q_{b_{k}}\right)=x_{\pi\left(b_{k}\right)}$ for all $k=1, \cdots, l$. Then

$$
\begin{aligned}
\left(\sigma_{x_{j}, H} \circ_{h} \sigma_{x_{i}, P}\right)(\gamma)= & \widehat{\sigma}_{x_{j}, H}\left[\sigma_{x_{i}, P}(\gamma)\right] \\
= & \widehat{\sigma}_{x_{j}, H}\left[\gamma\left(p_{1}, \cdots, p_{n}\right)\right] \\
= & \left.R_{n}^{n}\left(\sigma_{x_{j}, H}(\gamma), \widehat{\sigma}_{x_{j}, H}\left[p_{1}\right], \cdots, \widehat{\sigma}_{x_{j}, H}\left[p_{n}\right]\right)\right] \\
= & R_{n}^{n}\left(H, j-\operatorname{most}\left(p_{1}\right), \cdots, j-\operatorname{most}\left(p_{n}\right)\right) \\
= & \pi^{-1}(H), \\
& \text { since } I(H)=\left\{d_{1}, \cdots, d_{l}\right\} \text { and } j-\operatorname{most}\left(p_{d_{k}}\right)=x_{\pi^{-1}\left(d_{k}\right)} \\
& \text { for all } k=1, \cdots, l, \\
= & \pi^{-1}(\pi(F))=F=\sigma_{x_{i}, F}(\gamma) .
\end{aligned}
$$

Since $\left(\sigma_{x_{j}, H} \circ_{h} \sigma_{x_{i}, P}\right)(f)=x_{i}=\sigma_{x_{i}, F}(f)$, we have $\sigma_{x_{j}, H} \circ_{h} \sigma_{x_{i}, P}=\sigma_{x_{i}, F}$.

Similarly, we have $\sigma_{x_{i}, F} \circ_{h} \sigma_{x_{j}, Q}=\sigma_{x_{j}, H}$. Therefore $\sigma_{x_{i}, F} \mathcal{R} \sigma_{x_{j}, H}$.

Theorem 3.3. Let $\sigma_{x_{i}, F}, \sigma_{x_{j}, H} \in R_{X}$. Then $\sigma_{x_{i}, F} \mathcal{J} \sigma_{x_{j}, H}$ if and only if $|I(H)|=|I(F)|$.

Proof. Let $\sigma_{x_{i}, F} \mathcal{J} \sigma_{x_{j}, H}$ where $F=\gamma\left(s_{1}, \cdots, s_{n}\right), H=\gamma\left(h_{1}, \cdots, h_{n}\right) \in r F_{((m),(n))}\left(X_{n}\right)$. Then there are $\sigma_{u, G}, \sigma_{v, E}, \sigma_{p, O}, \sigma_{q, R} \in \operatorname{Relhyp}((m),(n))$ such that $\sigma_{x_{i}, F}=\sigma_{u, G} \circ_{h} \sigma_{x_{j}, H} \circ_{h}$ $\sigma_{v, E}$ and $\sigma_{x_{j}, H}=\sigma_{p, O} \circ_{h} \sigma_{x_{i}, F} \circ_{h} \sigma_{q, R}$ where $u, v, p, q \in W_{(m)}\left(X_{m}\right)$ and $E=\gamma\left(e_{1}, \cdots, e_{n}\right)$, $G=\gamma\left(g_{1}, \cdots, g_{n}\right), O=\gamma\left(o_{1}, \cdots, o_{n}\right), R=\gamma\left(r_{1}, \cdots, r_{n}\right) \in r F_{((m),(n))}\left(X_{n}\right)$. First of all, we consider $\sigma_{x_{j}, H} \circ_{h} \sigma_{v, E}$ and $\sigma_{x_{i}, F} \circ_{h} \sigma_{q, R}$. Then

$\left(\sigma_{x_{j}, H} \circ_{h} \sigma_{v, E}\right)(\gamma)=\widehat{\sigma}_{x_{j}, H}[E]=R_{n}^{n}\left(H, j-\operatorname{most}\left(e_{1}\right), \cdots, j-\operatorname{most}\left(e_{n}\right)\right)=\gamma\left(\widetilde{h}_{1}, \cdots, \widetilde{h}_{n}\right)$,

where $\widetilde{h}_{\iota}=S_{n}^{n}\left(h_{\iota}, j-\operatorname{most}\left(e_{1}\right), \cdots, j-\operatorname{most}\left(e_{n}\right)\right)$, for all $\iota=1, \cdots, n$, and

$$
\left(\sigma_{x_{i}, F} \circ_{h} \sigma_{q, R}\right)(\gamma)=\widehat{\sigma}_{x_{i}, F}[R]=R_{n}^{n}\left(F, i-\operatorname{most}\left(r_{1}\right), \cdots, i-\operatorname{most}\left(r_{n}\right)\right)=\gamma\left(\widetilde{s}_{1}, \cdots, \widetilde{s}_{n}\right),
$$


where $\widetilde{s}_{\iota}=S_{n}^{n}\left(s_{\iota}, i-\operatorname{most}\left(r_{1}\right), \cdots, i-\operatorname{most}\left(r_{n}\right)\right)$ for all $\iota=1, \cdots, n$. Next, we consider $\sigma_{x_{i}, F}=\sigma_{u, G} \circ_{h} \sigma_{x_{j}, H} \circ_{h} \sigma_{v, E}$. By (3.1), we have

$$
\begin{aligned}
F & =\left(\sigma_{x_{i}, F}\right)(\gamma) \\
& =\left(\sigma_{u, G} \circ_{h} \sigma_{x_{j}, H} \circ_{h} \sigma_{v, E}\right)(\gamma) \\
& =\widehat{\sigma}_{u, G}\left[\left(\sigma_{x_{j}, H} \circ_{h} \sigma_{v, E}\right)(\gamma)\right] \\
& =\widehat{\sigma}_{u, G}\left[\gamma\left(\widetilde{h}_{1}, \cdots, \widetilde{h}_{n}\right)\right] \\
& =R_{n}^{n}\left(G, \widehat{\sigma}_{u, G}\left[\widetilde{h}_{1}\right], \cdots, \widehat{\sigma}_{u, G}\left[\widetilde{h}_{n}\right]\right),
\end{aligned}
$$

where $s_{\iota}=S_{n}^{n}\left(g_{\iota}, \widehat{\sigma}_{u, G}\left[\widetilde{h}_{1}\right], \cdots, \widehat{\sigma}_{u, G}\left[\widetilde{h}_{n}\right]\right)$ for all $\iota=1, \cdots, n$.

Since $\sigma_{x_{i}, F} \in R_{X}$, there exists $k \in\{1, \cdots, m\}$ and some distinct $b_{1}^{\prime}, \cdots, b_{l}^{\prime} \in\{1, \cdots, n\}$ such that $k-\operatorname{most}\left(s_{b_{\rho}^{\prime}}\right)=x_{b_{\rho}}$ for all $\rho=1, \cdots, l$ where $I(F)=\left\{b_{1}, \cdots, b_{l}\right\}$. We obtain that

$$
x_{b_{\rho}}=k-\operatorname{most}\left(s_{b_{\rho}^{\prime}}\right)=S_{n}^{n}\left(k-\operatorname{most}\left(g_{b_{\rho}^{\prime}}\right), k-\operatorname{most}\left(\widehat{\sigma}_{u, G}\left[\widetilde{h}_{1}\right]\right), \cdots, k-\operatorname{most}\left(\widehat{\sigma}_{u, G}\left[\widetilde{h}_{n}\right]\right)\right)
$$

for all $\rho=1, \cdots, l$. Without loss of generality, for any $\rho=1, \cdots, l$, we can assume that $k-$ $\operatorname{most}\left(g_{b_{\rho}^{\prime}}\right)=x_{d_{\rho}^{\prime}}$ where $d_{\rho}^{\prime} \in I(G)$. Then $x_{b_{\rho}}=k-\operatorname{most}\left(\widehat{\sigma}_{u, G}\left[\widetilde{h}_{d_{\rho}^{\prime}}\right]\right)$ for all $\rho=1, \cdots, l$. Since $k-\operatorname{most}(u)$ exists, we can fix $k-\operatorname{most}(u)=x_{\alpha}$ for some $\alpha=1, \cdots, m$. By (3.1) and Lemma 2.1, we have $x_{b_{\rho}}=k-\operatorname{most}\left(\widehat{\sigma}_{u, G}\left[\widetilde{h}_{d_{\rho}^{\prime}}\right]\right)=\alpha-\operatorname{most}\left(\widetilde{h}_{d_{\rho}^{\prime}}\right)=S_{n}^{n}\left(\alpha-\operatorname{most}\left(h_{d_{\rho}^{\prime}}\right), j-\right.$ $\left.\operatorname{most}\left(e_{1}\right), \cdots, j-\operatorname{most}\left(e_{n}\right)\right)$ for all $\rho=1, \cdots, l$. So, if $\alpha-\operatorname{most}\left(h_{d_{\rho}^{\prime}}\right)=x_{d_{\rho}}$ where $d_{\rho} \in I(H)$ then $x_{b_{\rho}}=j-\operatorname{most}\left(e_{d_{\rho}}\right)$. Hence, for any $b_{\rho} \in I(F)$, there exists $d_{\rho} \in I(H)$ such that $x_{b_{\rho}}=j-$ $\operatorname{most}\left(e_{d_{\rho}}\right)$. If some pair of distinct elements $b_{\rho}, b_{\eta} \in I(F)$ which arise $x_{d_{\rho}}, x_{d_{\eta}} \in\left\{x_{d_{1}}, \cdots, x_{d_{l}}\right\}$ such that $d_{\rho}=d_{\eta}$, then $x_{b_{\rho}}=j-\operatorname{most}\left(e_{d_{\rho}}\right)=j-\operatorname{most}\left(e_{d_{\eta}}\right)=x_{b_{\eta}}$ which is a contradiction with $b_{\rho} \neq b_{\eta} \in I(F)$. So we obtain the set of all distinct elements $\left\{d_{1}, \cdots, d_{l}\right\} \subseteq I(H)$. Thus $|I(F)|=\left|\left\{d_{1}, \cdots, d_{l}\right\}\right| \leq|I(H)|$. Similarly, by using (3.2), we have $|I(H)| \leq|I(F)|$. Therefore $|I(H)|=|I(F)|$.

Conversely, assume that the condition holds. Let $\sigma_{x_{i}, F}, \sigma_{x_{j}, H} \in R_{X}$ where $F=\gamma\left(s_{1}, \cdots, s_{n}\right)$ and $H=\gamma\left(h_{1}, \cdots, h_{n}\right)$ with $I(F)=\left\{b_{1}, \cdots, b_{l}\right\}$ and $I(H)=\left\{d_{1}, \cdots, d_{l}\right\}$. There exist $\alpha, \beta \in$ $\{1, \cdots, m\}$ and subsets $\left\{b_{1}^{\prime}, \cdots, b_{l}^{\prime}\right\},\left\{d_{1}^{\prime}, \cdots, d_{l}^{\prime}\right\}$ of $\{1, \cdots, n\}$ such that $\alpha-\operatorname{most}\left(s_{b_{k}^{\prime}}\right)=x_{b_{k}}$ and $\beta-\operatorname{most}\left(h_{d_{k}^{\prime}}\right)=x_{d_{k}}$ for all $k=1, \cdots, l$. We will show that $\sigma_{x_{i}, F} \mathcal{J} \sigma_{x_{j}, H}$. First, we define $\pi:\left\{b_{1}, \cdots, b_{l}\right\} \rightarrow\left\{d_{1}^{\prime}, \cdots, d_{l}^{\prime}\right\}$ by $\pi\left(b_{k}\right)=d_{k}^{\prime}$, for all $k=1, \cdots, l$, and define $\phi:\left\{d_{1}, \cdots, d_{l}\right\} \rightarrow\left\{b_{1}^{\prime}, \cdots, b_{l}^{\prime}\right\}$ by $\phi\left(d_{k}\right)=b_{k}^{\prime}$, for all $k=1, \cdots, l$. Then $\pi, \phi$ are bijective. Choose $\sigma_{x_{\beta}, \pi(F)}, \sigma_{x_{\alpha}, \phi(H)}, \sigma_{x_{i}, E}, \sigma_{x_{j}, R} \in \operatorname{Relhyp}((m),(n))$ where $E=\gamma\left(e_{1}, \cdots, e_{n}\right)$, $R=\gamma\left(r_{1}, \cdots, r_{n}\right) \in r F_{((m),(n))}\left(X_{n}\right)$ such that $j-\operatorname{most}\left(e_{d_{k}}\right)=x_{b_{k}}$ and $i-\operatorname{most}\left(r_{b_{k}}\right)=x_{d_{k}}$ for all $k=1, \cdots, l$. Next, we want to show that $\sigma_{x_{\beta}, \pi(F)} \circ_{h} \sigma_{x_{j}, H} \circ_{h} \sigma_{x_{i}, E}=\sigma_{x_{i}, F}$ and $\sigma_{x_{\alpha}, \phi(H)}{ }^{\circ} \sigma_{x_{i}, F} \circ_{h} \sigma_{x_{j}, R}=\sigma_{x_{j}, H}$. For convenience, we consider

$$
\left(\sigma_{x_{j}, H} \circ_{h} \sigma_{x_{i}, E}\right)(\gamma)=\widehat{\sigma}_{x_{j}, H}\left[\sigma_{x_{i}, E}(\gamma)\right]
$$




$$
\begin{aligned}
& =\widehat{\sigma}_{x_{j}, H}\left[\gamma\left(e_{1}, \cdots, e_{n}\right)\right] \\
& =R_{n}^{n}\left(\sigma_{x_{j}, H}(\gamma), \widehat{\sigma}_{x_{j}, H}\left[e_{1}\right], \cdots, \widehat{\sigma}_{x_{j}, H}\left[e_{n}\right]\right) \\
& =R_{n}^{n}\left(H, j-\operatorname{most}\left(e_{1}\right), \cdots, j-\operatorname{most}\left(e_{n}\right)\right) \\
& =\gamma\left(\widetilde{h}_{1}, \cdots, \widetilde{h}_{n}\right)
\end{aligned}
$$

where $\widetilde{h}_{p}=S_{n}^{n}\left(h_{p}, j-\operatorname{most}\left(e_{1}\right), \cdots, j-\operatorname{most}\left(e_{n}\right)\right)$ for all $p=1, \cdots, n$.

Since $\beta-\operatorname{most}\left(h_{d_{k}^{\prime}}\right)=x_{d_{k}}$ and $j-\operatorname{most}\left(e_{d_{k}}\right)=x_{b_{k}}$, for all $k=1, \cdots, l$., we have

$$
\begin{aligned}
\beta-\operatorname{most}\left(\widetilde{h}_{d_{k}^{\prime}}\right) & =\beta-\operatorname{most}\left(S_{n}^{n}\left(h_{d_{k}^{\prime}}, j-\operatorname{most}\left(e_{1}\right), \cdots, j-\operatorname{most}\left(e_{n}\right)\right)\right) \\
& =S_{n}^{n}\left(\beta-\operatorname{most}\left(h_{d_{k}^{\prime}}\right), j-\operatorname{most}\left(e_{1}\right), \cdots, j-\operatorname{most}\left(e_{n}\right)\right) \\
& =S_{n}^{n}\left(x_{d_{k}}, j-\operatorname{most}\left(e_{1}\right), \cdots, j-\operatorname{most}\left(e_{n}\right)\right) \\
& =j-\operatorname{most}\left(e_{d_{k}}\right)=x_{b_{k}}
\end{aligned}
$$

for all $k=1, \cdots, l$. Similarly, if we consider $\sigma_{x_{i}, F} \circ_{h} \sigma_{x_{j}, R}$ then we have $\left(\sigma_{x_{i}, F} \circ_{h} \sigma_{x_{j}, R}\right)(\gamma)=$ $\gamma\left(\widetilde{s}_{1}, \cdots, \widetilde{s}_{n}\right)$ with $\alpha-\operatorname{most}\left(\widetilde{s}_{b_{k}^{\prime}}\right)=x_{d_{k}}$ for all $k=1, \cdots, l$.

By definition of bijective maps $\pi$ and $\phi$, we obtain $\beta-\operatorname{most}\left(\widetilde{h}_{d_{k}^{\prime}}\right)=x_{b_{k}}=x_{\pi^{-1}\left(d_{k}^{\prime}\right)}$ and $\alpha-$ $\operatorname{most}\left(\widetilde{s}_{b_{k}^{\prime}}\right)=x_{d_{k}}=x_{\phi^{-1}\left(b_{k}^{\prime}\right)}$ for all $k=1, \cdots, l$. Next, we consider

$$
\begin{aligned}
\left(\sigma_{x_{\beta}, \pi(F)} \circ_{h} \sigma_{x_{j}, H} \circ_{h} \sigma_{x_{i}, E}\right)(\gamma)= & \widehat{\sigma}_{x_{\beta}, \pi(F)}\left[\left(\sigma_{x_{j}, H} \circ_{h} \sigma_{x_{i}, E}\right)(\gamma)\right] \\
= & \widehat{\sigma}_{x_{\beta}, \pi(F)}\left[\gamma\left(\widetilde{h}_{1}, \cdots, \widetilde{h}_{n}\right)\right] \\
= & R_{n}^{n}\left(\sigma_{x_{\beta}, \pi(F)}(\gamma), \widehat{\sigma}_{x_{\beta}, \pi(F)}\left[\widetilde{h}_{1}\right], \cdots, \widehat{\sigma}_{x_{\beta}, \pi(F)}\left[\widetilde{h}_{n}\right]\right) \\
= & R_{n}^{n}\left(\pi(F), \beta-\operatorname{most}\left(\widetilde{h}_{1}\right), \cdots, \beta-\operatorname{most}\left(\widetilde{h}_{n}\right)\right) \\
= & \pi^{-1}(\pi(F)), \\
& \operatorname{since} I(\pi(F))=\left\{d_{1}^{\prime}, \cdots, d_{l}^{\prime}\right\}, \\
= & F=\sigma_{x_{i}, F}(\gamma) .
\end{aligned}
$$

It is obvious that $\left(\sigma_{x_{\beta}, \pi(F)}{ }^{\circ}{ }_{h} \sigma_{x_{j}, H}{ }^{\circ} \sigma_{x_{i}, E}\right)(f)=x_{i}=\sigma_{x_{i}, F}(f)$. So $\sigma_{x_{\beta}, \pi(F)}{ }^{\circ}{ }_{h} \sigma_{x_{j}, H^{\circ} h} \sigma_{x_{i}, E}=$ $\sigma_{x_{i}, F}$. Similarly, $\sigma_{x_{\alpha}, \phi(H)}{ }^{\circ} \sigma_{x_{i}, F} \circ_{h} \sigma_{x_{j}, R}=\sigma_{x_{j}, H}$. Therefore $\sigma_{x_{i}, F} \mathcal{J} \sigma_{x_{j}, H}$.

Theorem 3.4. In $R_{X}, \mathcal{J}=\mathcal{D}$.

Proof. It is obvious that $\mathcal{D} \subseteq \mathcal{J}$. We will show that $\mathcal{J} \subseteq \mathcal{D}$. Let $\sigma_{x_{i}, F} \mathcal{J} \sigma_{x_{j}, H}$ where $\sigma_{x_{i}, F}$, $\sigma_{x_{j}, H} \in R_{X}$ with $F=\gamma\left(s_{1}, \cdots, s_{n}\right), H=\gamma\left(h_{1}, \cdots, h_{n}\right) \in r F_{((m),(n))}\left(X_{n}\right)$. By Theorem 3.3, we obtain that $|I(H)|=|I(F)|$. We want to show that $\sigma_{x_{i}, F} \mathcal{D} \sigma_{x_{j}, H}$, i.e. there exists $\sigma_{p, G} \in \operatorname{Relhyp}((m),(n))$ such that $\sigma_{x_{i}, F} \mathcal{R} \sigma_{p, G}$ and $\sigma_{p, G} \mathcal{L} \sigma_{x_{j}, H}$. Since $|I(H)|=|I(F)|$, we assume that $I(F)=\left\{b_{1}, \cdots, b_{l}\right\}$ and $I(H)=\left\{d_{1}, \cdots, d_{l}\right\}$. Define $\pi:\left\{b_{1}, \cdots, b_{l}\right\} \rightarrow$ $\left\{d_{1}, \cdots, d_{l}\right\}$ by $\pi\left(b_{k}\right)=d_{k}$ for all $k=1, \cdots, l$. Then $\pi$ is a bijective map. Choose $\sigma_{p, G} \in$ 
Relhyp $((m),(n))$ with $p=x_{i}$ and $G=\pi(F)$. Then $I(G)=I(\pi(F))=\left\{\pi\left(b_{1}\right), \cdots, \pi\left(b_{l}\right)\right\}=$ $\left\{d_{1}, \cdots, d_{l}\right\}=I(H)$. By Theorem 3.1, we obtain that $\sigma_{p, G} \mathcal{L} \sigma_{x_{j}, H}$. Next, we prove that $\sigma_{x_{i}, F} \mathcal{R} \sigma_{p, G}$. Choose $\sigma_{x_{i}, R}, \sigma_{x_{i}, E} \in \operatorname{Relhyp}((m),(n))$ where $R=\gamma\left(r_{1}, \cdots, r_{n}\right), E=$ $\gamma\left(e_{1}, \cdots, e_{n}\right) \in r F_{((m),(n))}\left(X_{n}\right)$ such that $i-\operatorname{most}\left(r_{b_{k}}\right)=x_{\pi\left(b_{k}\right)}$ and $i-\operatorname{most}\left(e_{d_{k}}\right)=x_{\pi^{-1}\left(d_{k}\right)}$ for all $k=1, \cdots, l$. Then

$$
\begin{aligned}
\left(\sigma_{p, G} \circ_{h} \sigma_{x_{i}, E}\right)(\gamma)= & \widehat{\sigma}_{x_{i}, \pi(F)}\left[\sigma_{x_{i}, E}(\gamma)\right] \\
= & \widehat{\sigma}_{x_{i}, \pi(F)}\left[\gamma\left(e_{1}, \cdots, e_{n}\right)\right] \\
= & R_{n}^{n}\left(\sigma_{x_{i}, \pi(F)}(\gamma), \widehat{\sigma}_{x_{i}, \pi(F)}\left[e_{1}\right], \cdots, \widehat{\sigma}_{x_{i}, \pi(F)}\left[e_{n}\right]\right) \\
= & R_{n}^{n}\left(\pi(F), i-\operatorname{most}\left(e_{1}\right), \cdots, i-\operatorname{most}\left(e_{n}\right)\right) \\
= & \pi^{-1}(\pi(F)) \\
& \operatorname{since} I(\pi(F))=\left\{d_{1}, \cdots, d_{l}\right\} \\
= & F=\sigma_{x_{i}, F}(\gamma) \\
\left(\sigma_{x_{i}, F} \circ_{h} \sigma_{x_{i}, R}\right)(\gamma)= & \widehat{\sigma}_{x_{i}, F}\left[\sigma_{x_{i}, R}(\gamma)\right] \\
= & \widehat{\sigma}_{x_{i}, F}\left[\gamma\left(r_{1}, \cdots, r_{n}\right)\right] \\
= & R_{n}^{n}\left(\sigma_{x_{i}, F}(\gamma), \widehat{\sigma}_{x_{i}, F}\left[r_{1}\right], \cdots, \widehat{\sigma}_{x_{i}, F}\left[r_{n}\right]\right) \\
= & R_{n}^{n}\left(F, i-\operatorname{most}\left(r_{1}\right), \cdots, i-\operatorname{most}\left(r_{n}\right)\right) \\
= & \pi(F), \\
& \operatorname{since} i-\operatorname{most}\left(r_{b_{k}}\right)=x_{\pi\left(b_{k}\right)} \text { for all } k=1, \cdots, l, \\
= & G=\sigma_{p, G}(\gamma) .
\end{aligned}
$$

It is obvious that $\left(\sigma_{p, G} \circ_{h} \sigma_{x_{i}, E}\right)(f)=x_{i}=\sigma_{x_{i}, F}(f)$ and $\left(\sigma_{x_{i}, F} \circ_{h} \sigma_{x_{i}, R}\right)(f)=x_{i}=p=$ $\sigma_{p, G}(f)$. So $\sigma_{p, G} \circ_{h} \sigma_{x_{i}, E}=\sigma_{x_{i}, F}$ and $\sigma_{x_{i}, F} \circ_{h} \sigma_{x_{i}, R}=\sigma_{p, G}$. It follows that $\sigma_{x_{i}, F} \mathcal{R} \sigma_{p, G}$. Therefore $\sigma_{x_{i}, F} \mathcal{D} \sigma_{x_{j}, H}$ and the proof is complete.

Theorem 3.5. Let $\sigma_{x_{i}, F}, \sigma_{x_{j}, H} \in R_{X}$. Then $\sigma_{x_{i}, F} \mathcal{H} \sigma_{x_{j}, H}$ if and only if

(i) $I(H)=I(F)$

(ii) $i=j$ and $H=\pi(F)$ where $\pi$ is a bijective map on $I(F)$.

Proof. The proof is completed by Theorem 3.1 and Theorem 3.2.

Next, we study Green's relations when one element is in $R_{X}$ and another one is in $R_{T}$. We obtain that $R_{X}$ and $R_{T}$ are not related under Green's relations. 
Theorem 3.6. Any relational hypersubstitution $\sigma_{x_{i}, F} \in R_{X}$ is not $\mathcal{R}$-, $\mathcal{L}$-, $\mathcal{H}$-, $\mathcal{D}$ - and $\mathcal{J}$-related to any relational hypersubstitution in $R_{T}$.

Proof. Let $\sigma_{x_{i}, F} \in R_{X}$ and $\sigma_{w, H} \in R_{T}$ where $w \in W_{(m)}\left(X_{m}\right) \backslash X_{m}$. Consider $\mathcal{J}$-class, assume that $\sigma_{x_{i}, F} \mathcal{J} \sigma_{w, H}$. There are $\sigma_{u, G}, \sigma_{v, E}, \sigma_{p, O}, \sigma_{q, R} \in \operatorname{Relhyp}((m),(n))$ such that $\sigma_{x_{i}, F}=$ $\sigma_{u, G} \circ_{h} \sigma_{w, H} \circ_{h} \sigma_{v, E}$ and $\sigma_{w, H}=\sigma_{p, O} \circ_{h} \sigma_{x_{i}, F} \circ_{h} \sigma_{q, R}$. Then

$$
\begin{aligned}
w & =\sigma_{w, H}(f) \\
& =\left(\sigma_{p, O} \circ_{h} \sigma_{x_{i}, F} \circ_{h} \sigma_{q, R}\right)(f) \\
& =\widehat{\sigma}_{p, O}\left[\widehat{\sigma}_{x_{i}, F}\left[\sigma_{q, R}(f)\right]\right] \\
& =\widehat{\sigma}_{p, O}\left[\widehat{\sigma}_{x_{i}, F}[q]\right] \\
& =\widehat{\sigma}_{p, O}[i-\operatorname{most}(q)] \\
& =i-\operatorname{most}(q) \in X_{m} .
\end{aligned}
$$

This contradicts with $w \in W_{(m)}\left(X_{m}\right) \backslash X_{m}$. So $\left(\sigma_{x_{i}, F}, \sigma_{w, H}\right) \notin \mathcal{J}$ for all $\sigma_{x_{i}, F} \in R_{X}$ and $\sigma_{w, H} \in R_{T}$. Since $\mathcal{H} \subseteq \mathcal{R} \subseteq \mathcal{D} \subseteq \mathcal{J}$ and $\mathcal{H} \subseteq \mathcal{L} \subseteq \mathcal{D} \subseteq \mathcal{J}$, it also implies that $\left(\sigma_{x_{i}, F}, \sigma_{w, H}\right) \notin$ $\mathcal{R}, \mathcal{L}, \mathcal{H}$ and $\mathcal{D}$ for all $\sigma_{x_{i}, F} \in R_{X}$ and $\sigma_{w, H} \in R_{T}$. Therefore, we can conclude that any $\sigma_{x_{i}, F} \in$ $R_{X}$ is not $\mathcal{R}-, \mathcal{L}-, \mathcal{H}-, \mathcal{D}$ - and $\mathcal{J}$-related to any other relational hypersubstitution in $R_{T}$.

Finally, we characterize the Green's relations on $R_{T}$ as follows.

Theorem 3.7. Let $\sigma_{t, F}, \sigma_{w, H} \in R_{T}$. Then $\sigma_{t, F} \mathcal{L} \sigma_{w, H}$ if and only if $I(w)=I(t)$ and $I(H)=$ $I(F)$.

Proof. Let $\sigma_{t, F} \mathcal{L} \sigma_{w, H}$. There exist $\sigma_{u, G}, \sigma_{v, O} \in \operatorname{Relhyp}((m),(n))$ such that $\sigma_{t, F}=\sigma_{u, G} \circ_{h}$ $\sigma_{w, H}$ and $\sigma_{w, H}=\sigma_{v, O} \circ_{h} \sigma_{t, F}$. Then $t=\sigma_{t, F}(f)=\left(\sigma_{u, G} \circ_{h} \sigma_{w, H}\right)(f)$ and $w=\sigma_{w, H}(f)=$ $\left(\sigma_{v, O} \circ_{h} \sigma_{t, F}\right)(f)$. By Lemma 2.2, we obtain that $I(t) \subseteq I(w)$ and $I(w) \subseteq I(t)$. It follows that $I(w)=I(t)$. On the other hand, $F=\sigma_{t, F}(\gamma)=\left(\sigma_{u, G} \circ_{h} \sigma_{w, H}\right)(\gamma)$ and $H=\sigma_{w, H}(\gamma)=$ $\left(\sigma_{v, O} \circ_{h} \sigma_{t, F}\right)(\gamma)$. By Lemma 2.2, we have $I(F) \subseteq I(H)$ and $I(H) \subseteq I(F)$. So $I(H)=I(F)$.

Conversely, assume that the conditions hold. Let $\sigma_{w, H}, \sigma_{t, F} \in R_{T}$ where $w=f\left(w_{1}, \cdots, w_{m}\right)$, $t=f\left(t_{1}, \cdots, t_{m}\right) \in W_{(m)}\left(X_{m}\right)$ and $H=\gamma\left(h_{1}, \cdots, h_{n}\right), F=\gamma\left(s_{1}, \cdots, s_{n}\right) \in r F_{((m),(n))}\left(X_{n}\right)$ with $I(w)=\left\{a_{1}, \cdots, a_{k}\right\}=I(t)$ and $I(H)=\left\{b_{1}, \cdots, b_{l}\right\}=I(F)$. We will show that $\sigma_{t, F} \mathcal{L} \sigma_{w, H}$, i.e. there exist $\sigma_{u, G}, \sigma_{v, O} \in \operatorname{Relhyp}((m),(n))$ such that $\sigma_{u, G} \circ_{h} \sigma_{t, F}=\sigma_{w, H}$ and $\sigma_{v, O} \circ_{h} \sigma_{w, H}=\sigma_{t, F}$. Since $\sigma_{t, F}, \sigma_{w, H} \in R_{T}$, there exist subsets $\left\{a_{1}^{\prime}, \cdots, a_{k}^{\prime}\right\},\left\{\widetilde{a}_{1}, \cdots, \widetilde{a}_{k}\right\}$ of $\{1, \cdots, m\}$ and subsets $\left\{b_{1}^{\prime}, \cdots, b_{l}^{\prime}\right\},\left\{\widetilde{b}_{1}, \cdots, \widetilde{b}_{l}\right\}$ of $\{1, \cdots, n\}$ such that $w_{a_{i}^{\prime}}=x_{a_{i}}=t_{\widetilde{a}_{i}}$ and $h_{b_{j}^{\prime}}=x_{b_{j}}=s_{\widetilde{b}_{j}}$ for all $i=1, \cdots, k$ and $j=1, \cdots, l$. Define $\pi_{1}:\left\{a_{1}, \cdots, a_{k}\right\} \rightarrow$ $\left\{\widetilde{a}_{1}, \cdots, \widetilde{a}_{k}\right\}$ by $\pi_{1}\left(a_{i}\right)=\widetilde{a}_{i}$ for all $i=1, \cdots, k$,

$\pi_{2}:\left\{a_{1}, \cdots, a_{k}\right\} \rightarrow\left\{a_{1}^{\prime}, \cdots, a_{k}^{\prime}\right\}$ by $\pi_{2}\left(a_{i}\right)=a_{i}^{\prime}$ for all $i=1, \cdots, k$, 
$\phi_{1}:\left\{b_{1}, \cdots, b_{l}\right\} \rightarrow\left\{\widetilde{b}_{1}, \cdots, \widetilde{b}_{l}\right\}$ by $\phi_{1}\left(b_{j}\right)=\widetilde{b}_{j}$ for all $j=1, \cdots, l$ and

$\phi_{2}:\left\{b_{1}, \cdots, b_{l}\right\} \rightarrow\left\{b_{1}^{\prime}, \cdots, b_{l}^{\prime}\right\}$ by $\phi_{2}\left(b_{j}\right)=b_{j}^{\prime}$ for all $j=1, \cdots, l$.

Then $\pi_{1}, \pi_{2}, \phi_{1}$ and $\phi_{2}$ are bijective. First, consider the mapping $\pi_{1}$, we have $a_{i}=\pi_{1}^{-1}\left(\widetilde{a}_{i}\right)$ for all $i=1, \cdots, k$. Then $t_{\widetilde{a}_{i}}=x_{a_{i}}=x_{\pi_{1}^{-1}\left(\widetilde{a}_{i}\right)}$ for all $i=1, \cdots, k$. Similarly, we have $w_{a_{i}^{\prime}}=x_{\pi_{2}^{-1}\left(a_{i}^{\prime}\right)}$, $s_{\widetilde{b}_{j}}=x_{\phi_{1}^{-1}\left(\widetilde{b}_{j}\right)}$ and $h_{b_{j}^{\prime}}=x_{\phi_{2}^{-1}\left(b_{j}^{\prime}\right)}$ for all $i=1, \cdots, k$ and $j=1, \cdots, l$.

Choose $\sigma_{u, G}, \sigma_{v, O} \in \operatorname{Relhyp}((m),(n))$ with $u=\pi_{1}(w), v=\pi_{2}(t), G=\phi_{1}(H)$ and $O=\phi_{2}(F)$. Then $I(u)=\left\{\widetilde{a}_{1}, \cdots, \widetilde{a}_{k}\right\}, I(v)=\left\{a_{1}^{\prime}, \cdots, a_{k}^{\prime}\right\}, I(G)=\left\{\widetilde{b}_{1}, \cdots, \widetilde{b}_{l}\right\}$ and $I(O)=\left\{b_{1}^{\prime}, \cdots, b_{l}^{\prime}\right\}$. We have

$$
\begin{aligned}
& \left(\sigma_{u, G} \circ_{h} \sigma_{t, F}\right)(f)=\widehat{\sigma}_{u, G}\left[\sigma_{t, F}(f)\right] \\
& =\widehat{\sigma}_{u, G}\left[f\left(t_{1}, \cdots, t_{m}\right)\right] \\
& =S_{m}^{m}\left(u, \widehat{\sigma}_{u, G}\left[t_{1}\right], \cdots, \widehat{\sigma}_{u, G}\left[t_{m}\right]\right) \text {, } \\
& \text { since } \widehat{\sigma}_{u, G}\left[t_{\widetilde{a}_{i}}\right]=x_{\pi_{1}^{-1}\left(\widetilde{a}_{i}\right)} \text { for all } i=1, \cdots, k \text {, } \\
& =\pi_{1}^{-1}(u)=\pi_{1}^{-1}\left(\pi_{1}(w)\right)=w=\sigma_{w, H}(f) \text {, } \\
& \left(\sigma_{u, G} \circ_{h} \sigma_{t, F}\right)(\gamma)=\widehat{\sigma}_{u, G}\left[\sigma_{t, F}(\gamma)\right] \\
& =\widehat{\sigma}_{u, G}\left[\gamma\left(s_{1}, \cdots, s_{n}\right)\right] \\
& =R_{n}^{n}\left(G, \widehat{\sigma}_{u, G}\left[s_{1}\right], \cdots, \widehat{\sigma}_{u, G}\left[s_{n}\right]\right) \text {, } \\
& \text { since } \widehat{\sigma}_{u, G}\left[s_{\widetilde{b}_{j}}\right]=x_{\phi_{1}^{-1}\left(\widetilde{b}_{j}\right)} \text { for all } j=1, \cdots, l \text {, } \\
& =\phi_{1}^{-1}(G)=\phi_{1}^{-1}\left(\phi_{1}(H)\right)=H=\sigma_{w, H}(\gamma) \text {. }
\end{aligned}
$$

Similarly, we have $\left(\sigma_{v, O} \circ_{h} \sigma_{w, H}\right)(f)=t=\sigma_{t, F}(f)$ and $\left(\sigma_{v, O} \circ_{h} \sigma_{w, H}\right)(\gamma)=F=\sigma_{t, F}(\gamma)$. It follows that $\sigma_{u, G}{ }^{\circ} \sigma_{t, F}=\sigma_{w, H}$ and $\sigma_{v, O}{ }^{\circ} \sigma_{w, H}=\sigma_{t, F}$. Therefore $\sigma_{t, F} \mathcal{L} \sigma_{w, H}$.

Theorem 3.8. Let $\sigma_{t, F}, \sigma_{w, H} \in R_{T}$. Then $\sigma_{t, F} \mathcal{R} \sigma_{w, H}$ if and only if

(i) $|I(w)|=|I(t)|$ and $|I(H)|=|I(F)|$,

(ii) $w=\pi(t)$ and $H=\phi(F)$ where $\pi: I(t) \rightarrow I(w)$ and $\phi: I(F) \rightarrow I(H)$ are bijective.

Proof. Let $\sigma_{t, F} \mathcal{R} \sigma_{w, H}$ where $t=f\left(t_{1}, \cdots, t_{m}\right), w=f\left(w_{1}, \cdots, w_{m}\right) \in W_{(m)}\left(X_{m}\right)$ and $F=$ $\gamma\left(s_{1}, \cdots, s_{n}\right), H=\gamma\left(h_{1}, \cdots, h_{n}\right) \in r F_{((m),(n))}\left(X_{n}\right)$. There exist $\sigma_{u, G}, \sigma_{v, O} \in \operatorname{Relhyp}((m),(n))$ such that

$$
\begin{gathered}
\sigma_{t, F}=\sigma_{w, H} \circ_{h} \sigma_{u, G}, \\
\sigma_{w, H}=\sigma_{t, F} \circ_{h} \sigma_{v, O} .
\end{gathered}
$$


where $u=f\left(u_{1}, \cdots, u_{m}\right), v=f\left(v_{1}, \cdots, v_{m}\right) \in W_{(m)}\left(X_{m}\right)$ and $G=\gamma\left(g_{1}, \cdots, g_{n}\right), O=$ $\gamma\left(o_{1}, \cdots, o_{n}\right) \in r F_{((m),(n))}\left(X_{n}\right)$. Consider

$$
\begin{aligned}
t & =\sigma_{t, F}(f) \\
& =\left(\sigma_{w, H} \circ_{h} \sigma_{u, G}\right)(f) \\
& =\widehat{\sigma}_{w, H}\left[\sigma_{u, G}(f)\right] \\
& =\widehat{\sigma}_{w, H}\left[f\left(u_{1}, \cdots, u_{m}\right)\right] \\
& =S_{m}^{m}\left(w, \widehat{\sigma}_{w, H}\left[u_{1}\right], \cdots, \widehat{\sigma}_{w, H}\left[u_{m}\right]\right)
\end{aligned}
$$

where $t_{p}=S_{m}^{m}\left(w_{p}, \widehat{\sigma}_{w, H}\left[u_{1}\right], \cdots, \widehat{\sigma}_{w, H}\left[u_{m}\right]\right)$ for all $p=1, \cdots, m$. Clearly, if $t_{p} \in X_{m}$ then $w_{p} \in X_{m}$. Since $\sigma_{t, F} \in R_{T}$, there are all distinct $a_{1}^{\prime}, \cdots, a_{k}^{\prime} \in\{1, \cdots, m\}$ and $b_{1}^{\prime}, \cdots, b_{l}^{\prime} \in$ $\{1, \cdots, n\}$ such that $t_{a_{1}^{\prime}}=x_{a_{1}}, \cdots, t_{a_{k}^{\prime}}=x_{a_{k}}$ and $s_{b_{1}^{\prime}}=x_{b_{1}}, \cdots, s_{b_{l}^{\prime}}=x_{b_{l}}$ where $I(t)=$ $\left\{a_{1}, \cdots, a_{k}\right\}$ and $I(F)=\left\{b_{1}, \cdots, b_{l}\right\}$. So $w_{a_{1}^{\prime}}, \cdots, w_{a_{k}^{\prime}} \in X_{m}$. If $w_{a_{i}^{\prime}}=w_{a_{j}^{\prime}}=x_{d}$ for some $i \neq j$ then

$$
\begin{aligned}
x_{a_{i}}= & t_{a_{i}^{\prime}}=S_{m}^{m}\left(w_{a_{i}^{\prime}}, \widehat{\sigma}_{w, H}\left[u_{1}\right], \cdots, \widehat{\sigma}_{w, H}\left[u_{m}\right]\right) \\
& =\widehat{\sigma}_{w, H}\left[u_{d}\right]=S_{m}^{m}\left(w_{a_{j}^{\prime}}, \widehat{\sigma}_{w, H}\left[u_{1}\right], \cdots, \widehat{\sigma}_{w, H}\left[u_{m}\right]\right)=t_{a_{j}^{\prime}}=x_{a_{j}} .
\end{aligned}
$$

This contradicts with $x_{a_{i}} \neq x_{a_{j}}$ for all $i \neq j$. Thus $w_{a_{i}^{\prime}} \neq w_{a_{j}^{\prime}}$ for all $i \neq j \in\{1, \cdots, k\}$. It implies that $|I(t)|=\left|\left\{w_{a_{1}^{\prime}}, \cdots, w_{a_{k}^{\prime}}\right\}\right| \leq|I(w)|$. Similarly, by using (3.4), we have $|I(w)| \leq$ $|I(t)|$. So $|I(w)|=|I(t)|$. By using (3.3) and (3.4) again, we have $|I(H)|=|I(F)|$ and the proof of (i) is complete.

Next, we will show (ii). Let $I(w)=\left\{c_{1}, \cdots, c_{k}\right\}$ and $I(H)=\left\{d_{1}, \cdots, d_{l}\right\}$. Since $w_{a_{1}^{\prime}}, \ldots, w_{a_{k}^{\prime}}$ $\in X_{m}$ such that $w_{a_{i}^{\prime}} \neq w_{a_{j}^{\prime}}$ for all $i \neq j$, we can assume that $w_{a_{1}^{\prime}}=x_{c_{1}}, \cdots, w_{a_{k}^{\prime}}=x_{c_{k}}$. Then $x_{a_{i}}=t_{a_{i}^{\prime}}=S_{m}^{m}\left(w_{a_{i}^{\prime}}, \widehat{\sigma}_{w, H}\left[u_{1}\right], \cdots, \widehat{\sigma}_{w, H}\left[u_{m}\right]\right)=\widehat{\sigma}_{w, H}\left[u_{c_{i}}\right]$ for all $i=1, \cdots, k$. Since $w \in W_{(m)}\left(X_{m}\right) \backslash X_{m}$, it implies $u_{c_{i}}=x_{a_{i}}$ for all $i=1, \cdots, k$. Define a bijective map $\pi:\left\{a_{1}, \cdots, a_{k}\right\} \rightarrow\left\{c_{1}, \cdots, c_{k}\right\}$ by $\pi\left(a_{i}\right)=c_{i}$ for all $i=1, \cdots, k$. Then $u_{c_{i}}=x_{a_{i}}=x_{\pi^{-1}\left(c_{i}\right)}$ for all $i=1, \cdots, k$ and it implies that $\widehat{\sigma}_{w, H}\left[u_{c_{i}}\right]=x_{\pi^{-1}\left(c_{i}\right)}$ for all $i=1, \cdots, k$. Since $I(w)=\left\{c_{1}, \cdots, c_{k}\right\}$, we have $t=S_{m}^{m}\left(w, \widehat{\sigma}_{w, H}\left[u_{1}\right], \cdots, \widehat{\sigma}_{w, H}\left[u_{m}\right]\right)=\pi^{-1}(w)$. Therefore, $w=\pi(t)$ where $\pi$ is a bijection from $I(t)$ to $I(w)$. Similarly, $H=\phi(F)$ where $\phi$ is a bijection from $I(F)$ to $I(H)$.

Conversely, assume that the conditions hold. Let $\sigma_{t, F}, \sigma_{w, H} \in R_{t}$ with $I(t)=\left\{a_{1}, \cdots, a_{k}\right\}$, $I(w)=\left\{c_{1}, \cdots, c_{k}\right\}, I(F)=\left\{b_{1}, \cdots, b_{l}\right\}$ and $I(H)=\left\{d_{1}, \cdots, d_{l}\right\}$ such that $w=\pi(t)$ and $H=\phi(F)$ where $\pi: I(t) \rightarrow I(w)$ and $\phi: I(F) \rightarrow I(H)$ are bijective. We will show that $\sigma_{t, F} \mathcal{R} \sigma_{w, H}$, i.e. there exist $\sigma_{u, G}, \sigma_{v, O} \in \operatorname{Relhyp}((m),(n))$ such that $\sigma_{w, H} \circ_{h} \sigma_{u, G}=\sigma_{t, F}$ and $\sigma_{t, F} \circ_{h} \sigma_{v, O}=\sigma_{w, H}$.

Choose $\sigma_{u, G}, \sigma_{v, O} \in \operatorname{Relhyp}((m),(n))$ where $u=f\left(u_{1}, \cdots, u_{m}\right), v=f\left(v_{1}, \cdots, v_{m}\right) \in$ $W_{(m)}\left(X_{m}\right)$ and $G=\gamma\left(g_{1}, \cdots, g_{n}\right), O=\gamma\left(o_{1}, \cdots, o_{n}\right) \in r F_{((m),(n))}\left(X_{n}\right)$ such that $u_{c_{i}}=$ 
$x_{\pi^{-1}\left(c_{i}\right)}, v_{a_{i}}=x_{\pi\left(a_{i}\right)}, g_{d_{j}}=x_{\phi^{-1}\left(d_{j}\right)}$ and $o_{b_{j}}=x_{\phi\left(b_{j}\right)}$ for all $i=1, \cdots, k$ and $j=1, \cdots, l$. Then

$$
\begin{aligned}
\left(\sigma_{w, H} \circ_{h} \sigma_{u, G}\right)(f)= & \widehat{\sigma}_{w, H}\left[\sigma_{u, G}(f)\right] \\
= & \widehat{\sigma}_{w, H}\left[f\left(u_{1}, \cdots, u_{m}\right)\right] \\
= & S_{m}^{m}\left(w, \widehat{\sigma}_{w, H}\left[u_{1}\right], \cdots, \widehat{\sigma}_{w, H}\left[u_{m}\right]\right), \\
& \text { since } \widehat{\sigma}_{w, H}\left[u_{c_{i}}\right]=x_{\pi^{-1}\left(c_{i}\right)} \text { for all } i=1, \cdots, k, \\
= & \pi^{-1}(w)=\pi^{-1}(\pi(t))=t=\sigma_{t, F}(f), \\
\left(\sigma_{w, H} \circ_{h} \sigma_{u, G}\right)(\gamma)= & \widehat{\sigma}_{w, H}\left[\sigma_{u, G}(\gamma)\right] \\
= & \widehat{\sigma}_{w, H}\left[\gamma\left(g_{1}, \cdots, g_{n}\right)\right] \\
= & R_{n}^{n}\left(H, \widehat{\sigma}_{w, H}\left[g_{1}\right], \cdots, \widehat{\sigma}_{w, H}\left[g_{n}\right]\right), \\
& \operatorname{since} \widehat{\sigma}_{w, H}\left[g_{d_{j}}\right]=x_{\phi^{-1}\left(d_{j}\right)} \text { for all } j=1, \cdots, l, \\
= & \phi^{-1}(H)=\phi^{-1}(\phi(F))=F=\sigma_{t, F}(\gamma) .
\end{aligned}
$$

Similarly, $\left(\sigma_{t, F} \circ_{h} \sigma_{v, O}\right)(f)=S_{m}^{m}\left(t, \widehat{\sigma}_{t, F}\left[v_{1}\right], \cdots, \widehat{\sigma}_{t, F}\left[v_{m}\right]\right)=\pi(t)=w=\sigma_{w, H}(f)$ and $\left(\sigma_{t, F} \circ_{h} \sigma_{v, O}\right)(\gamma)=R_{n}^{n}\left(F, \widehat{\sigma}_{t, F}\left[o_{1}\right], \cdots, \widehat{\sigma}_{t, F}\left[o_{n}\right]\right)=\phi(F)=H=\sigma_{w, H}(\gamma)$. Hence $\sigma_{w, H} \circ_{h}$ $\sigma_{u, G}=\sigma_{t, F}$ and $\sigma_{t, F} \circ_{h} \sigma_{v, O}=\sigma_{w, H}$. Therefore $\sigma_{t, F} \mathcal{R} \sigma_{w, H}$.

Theorem 3.9. Let $\sigma_{t, F}, \sigma_{w, H} \in R_{T}$. Then $\sigma_{t, F} \mathcal{J} \sigma_{w, H}$ if and only if $|I(w)|=|I(t)|$ and $|I(H)|=|I(F)|$.

Proof. Let $\sigma_{t, F} \mathcal{J} \sigma_{w, H}$. There are $\sigma_{u, G}, \sigma_{v, E}, \sigma_{p, O}, \sigma_{q, R} \in \operatorname{Relhyp}((m),(n))$ such that

$$
\begin{aligned}
\sigma_{t, F} & =\sigma_{u, G} \circ_{h} \sigma_{w, H} \circ_{h} \sigma_{v, E}, \\
\sigma_{w, H} & =\sigma_{p, O} \circ_{h} \sigma_{t, F} \circ_{h} \sigma_{q, R} .
\end{aligned}
$$

where $u=f\left(u_{1}, \cdots, u_{m}\right), v=f\left(v_{1}, \cdots, v_{m}\right) \in W_{(m)}\left(X_{m}\right)$ and $G=\gamma\left(g_{1}, \cdots, g_{n}\right), E=$ $\gamma\left(e_{1}, \cdots, e_{n}\right) \in r F_{((m),(n))}\left(X_{n}\right)$. First, we consider (3.5). For convenience, let $\left(\sigma_{w, H} \circ_{h} \sigma_{v, E}\right)(f)=\widehat{\sigma}_{w, H}[v]=S_{m}^{m}\left(w, \widehat{\sigma}_{w, H}\left[v_{1}\right], \cdots, \widehat{\sigma}_{w, H}\left[v_{m}\right]\right)=f\left(\widetilde{w}_{1}, \cdots, \widetilde{w}_{m}\right)$ where $\widetilde{w}_{j}=S_{m}^{m}\left(w_{j}, \widehat{\sigma}_{w, H}\left[v_{1}\right], \cdots, \widehat{\sigma}_{w, H}\left[v_{m}\right]\right)$ for all $j=1, \cdots, m$. Then

$$
\begin{aligned}
t & =\sigma_{t, F}(f) \\
& =\left(\sigma_{u, G} \circ_{h} \sigma_{w, H} \circ_{h} \sigma_{v, E}\right)(f) \\
& =\widehat{\sigma}_{u, G}\left[\left(\sigma_{w, H} \circ_{h} \sigma_{v, E}\right)(f)\right] \\
& =\widehat{\sigma}_{u, G}\left[f\left(\widetilde{w}_{1}, \cdots, \widetilde{w}_{m}\right)\right] \\
& =S_{m}^{m}\left(\sigma_{u, G}(f), \widehat{\sigma}_{u, G}\left[\widetilde{w}_{1}\right], \cdots, \widehat{\sigma}_{u, G}\left[\widetilde{w}_{m}\right]\right)
\end{aligned}
$$




$$
=S_{m}^{m}\left(u, \widehat{\sigma}_{u, G}\left[\widetilde{w}_{1}\right], \cdots, \widehat{\sigma}_{u, G}\left[\widetilde{w}_{m}\right]\right)
$$

So $t_{j}=S_{m}^{m}\left(u_{j}, \widehat{\sigma}_{u, G}\left[\widetilde{w}_{1}\right], \cdots, \widehat{\sigma}_{u, G}\left[\widetilde{w}_{m}\right]\right)$ for all $j=1, \cdots, m$. We know that if $t_{j} \in X_{m}$ then $u_{j} \in X_{m}$. Since $\sigma_{t, F} \in R_{T}$, there exist all distinct $a_{1}^{\prime}, \cdots, a_{k}^{\prime} \in\{1, \cdots, m\}$ and $b_{1}^{\prime}, \cdots, b_{l}^{\prime} \in$ $\{1, \cdots, n\}$ such that $t_{a_{1}^{\prime}}=x_{a_{1}}, \cdots, t_{a_{k}^{\prime}}=x_{a_{k}}$ and $s_{b_{1}^{\prime}}=x_{b_{1}}, \cdots, s_{b_{l}^{\prime}}=x_{b_{l}}$ where $I(t)=$ $\left\{a_{1}, \cdots, a_{k}\right\}$ and $I(F)=\left\{b_{1}, \cdots, b_{l}\right\}$. Similar to the proof of Theorem 3.8, we obtain that $u_{a_{1}^{\prime}}, \cdots, u_{a_{k}^{\prime}} \in X_{m}$ and $u_{a_{i}^{\prime}} \neq u_{a_{j}^{\prime}}$ for all $i \neq j$.

Without loss of generality, we can assume that $u_{a_{i}^{\prime}}=x_{\alpha_{i}}$ for all $i=1, \cdots, k$ (it also implies that $\alpha_{i} \neq \alpha_{j}$ for all $\left.i \neq j\right)$. Then $x_{a_{i}}=t_{a_{i}^{\prime}}=S_{m}^{m}\left(u_{a_{i}^{\prime}}, \widehat{\sigma}_{u, G}\left[\widetilde{w}_{1}\right], \cdots, \widehat{\sigma}_{u, G}\left[\widetilde{w}_{m}\right]\right)=\widehat{\sigma}_{u, G}\left[\widetilde{w}_{\alpha_{i}}\right]$ for all $i=1, \cdots, k$. Next, we show that $\widetilde{w}_{\alpha_{i}}=x_{a_{i}}$ for all $i=1, \cdots, k$. If $u=x_{\beta} \in X_{m}$ then, by (3.5), we have $t=\left(\sigma_{t, F}\right)(f)=\left(\sigma_{u, G} \circ_{h} \sigma_{w, H} \circ_{h} \sigma_{v, E}\right)(f)=\widehat{\sigma}_{x_{\beta}, G}\left[\left(\sigma_{w, H} \circ_{h} \sigma_{v, E}\right)(f)\right]=$ $\widehat{\sigma}_{x_{\beta}, G}\left[f\left(\widetilde{w}_{1}, \cdots, \widetilde{w}_{m}\right)\right]=\beta-\operatorname{most}\left(f\left(\widetilde{w}_{1}, \cdots, \widetilde{w}_{m}\right)\right)=\beta-\operatorname{most}\left(\widetilde{w}_{\beta}\right) \in X_{m}$, a contradiction with $t \in W_{(m)}\left(X_{m}\right) \backslash X_{m}$. So $u \in W_{(m)}\left(X_{m}\right) \backslash X_{m}$. Since $x_{a_{i}}=\widehat{\sigma}_{u, G}\left[\widetilde{w}_{\alpha_{i}}\right], \widetilde{w}_{\alpha_{i}}=x_{a_{i}}$ for all $i=1, \cdots, k$. Since $x_{a_{i}}=\widetilde{w}_{\alpha_{i}}=S_{m}^{m}\left(w_{\alpha_{i}}, \widehat{\sigma}_{w, H}\left[v_{1}\right], \cdots, \widehat{\sigma}_{w, H}\left[v_{m}\right]\right)$, we have $w_{\alpha_{1}}, \cdots, w_{\alpha_{k}} \in$ $X_{m}$ and are all distinct. So $|I(t)|=\left|\left\{w_{\alpha_{1}}, \cdots, w_{\alpha_{k}}\right\}\right| \leq|I(w)|$. By (3.6), we can show that $|I(w)| \leq|I(t)|$. Thus $|I(w)|=|I(t)|$. By (3.5) and (3.6) again, we have $|I(H)|=|I(F)|$.

Conversely, assume that the conditions hold. Let $\sigma_{t, F}, \sigma_{w, H} \in R_{T}$ with $I(t)=\left\{a_{1}, \cdots, a_{k}\right\}, I(w)=\left\{c_{1}, \cdots, c_{k}\right\}, I(F)=\left\{b_{1}, \cdots, b_{l}\right\}$ and $I(H)=\left\{d_{1}, \cdots, d_{l}\right\}$ where $t=f\left(t_{1}, \cdots, t_{m}\right), w=f\left(w_{1}, \cdots, w_{m}\right), F=\gamma\left(s_{1}, \cdots, s_{n}\right)$ and $H=\gamma\left(h_{1}, \cdots, h_{n}\right)$ such that $t_{a_{i}^{\prime}}=x_{a_{i}}, w_{c_{i}^{\prime}}=x_{c_{i}}, s_{b_{j}^{\prime}}=x_{b_{j}}$ and $h_{d_{j}^{\prime}}=x_{d_{j}}$ for all $i=1, \cdots, k$ and $j=1, \cdots, l$. We will show that $\sigma_{t, F} \mathcal{J} \sigma_{w, H}$. First, we define $\pi_{1}:\left\{a_{1}, \cdots, a_{k}\right\} \rightarrow\left\{c_{1}^{\prime}, \cdots, c_{k}^{\prime}\right\}$ by $\pi_{1}\left(a_{i}\right)=c_{i}^{\prime}$ for all $i=1, \cdots, k$,

$\pi_{2}:\left\{c_{1}, \cdots, c_{k}\right\} \rightarrow\left\{a_{1}^{\prime}, \cdots, a_{k}^{\prime}\right\}$ by $\pi_{2}\left(c_{i}\right)=a_{i}^{\prime}$ for all $i=1, \cdots, k$,

$\phi_{1}:\left\{b_{1}, \cdots, b_{l}\right\} \rightarrow\left\{d_{1}^{\prime}, \cdots, d_{l}^{\prime}\right\}$ by $\phi_{1}\left(b_{j}\right)=d_{j}^{\prime}$ for all $j=1, \cdots, l$ and

$\phi_{2}:\left\{d_{1}, \cdots, d_{l}\right\} \rightarrow\left\{b_{1}^{\prime}, \cdots, b_{l}^{\prime}\right\}$ by $\phi_{2}\left(d_{j}\right)=b_{j}^{\prime}$ for all $j=1, \cdots, l$.

Choose $\sigma_{\pi_{1}(t), \phi_{1}(F)}, \sigma_{\pi_{2}(w), \phi_{2}(H)}, \sigma_{u, E}, \sigma_{v, R} \in \operatorname{Relhyp}((m),(n))$ with $u=f\left(u_{1}, \cdots, u_{m}\right)$, $v=f\left(v_{1}, \cdots, v_{m}\right), E=\gamma\left(e_{1}, \cdots, e_{n}\right)$ and $R=\gamma\left(r_{1}, \cdots, r_{n}\right)$ such that $u_{c_{i}}=x_{a_{i}}, v_{a_{i}}=x_{c_{i}}$, $e_{d_{j}}=x_{b_{j}}$ and $r_{b_{j}}=x_{d_{j}}$ for all $i=1, \cdots, k, j=1, \cdots, l$. Next, we will show that $\sigma_{t, F}=$ $\sigma_{\pi_{1}(t), \phi_{1}(F)} \circ_{h} \sigma_{w, H} \circ_{h} \sigma_{u, E}$ and $\sigma_{w, H}=\sigma_{\pi_{2}(w), \phi_{2}(H)} \circ_{h} \sigma_{t, F} \circ_{h} \sigma_{v, R}$. For convenience, we consider

$$
\left(\sigma_{w, H} \circ_{h} \sigma_{u, E}\right)(f)=S_{m}^{m}\left(w, \widehat{\sigma}_{w, H}\left[u_{1}\right], \cdots, \widehat{\sigma}_{w, H}\left[u_{m}\right]\right)=f\left(\widetilde{w}_{1}, \cdots, \widetilde{w}_{m}\right)
$$

where $\widetilde{w}_{p}=S_{m}^{m}\left(w_{p}, \widehat{\sigma}_{w, H}\left[u_{1}\right], \cdots, \widehat{\sigma}_{w, H}\left[u_{m}\right]\right)$ for all $p=1, \cdots, m$,

$$
\left(\sigma_{t, F} \circ_{h} \sigma_{v, R}\right)(f)=S_{m}^{m}\left(t, \widehat{\sigma}_{t, F}\left[v_{1}\right], \cdots, \widehat{\sigma}_{t, F}\left[v_{m}\right]\right)=f\left(\widetilde{t}_{1}, \cdots, \widetilde{t}_{m}\right)
$$

where $\widetilde{t}_{p}=S_{m}^{m}\left(t_{p}, \widehat{\sigma}_{t, F}\left[v_{1}\right], \cdots, \widehat{\sigma}_{t, F}\left[v_{m}\right]\right)$ for all $p=1, \cdots, m$,

$$
\left(\sigma_{w, H} \circ_{h} \sigma_{u, E}\right)(\gamma)=R_{n}^{n}\left(H, \widehat{\sigma}_{w, H}\left[e_{1}\right], \cdots, \widehat{\sigma}_{w, H}\left[e_{n}\right]\right)=\gamma\left(\widetilde{h}_{1}, \cdots, \widetilde{h}_{n}\right)
$$


where $\widetilde{h}_{p}=S_{n}^{n}\left(h_{p}, \widehat{\sigma}_{w, H}\left[e_{1}\right], \cdots, \widehat{\sigma}_{w, H}\left[e_{m}\right]\right)$ for all $p=1, \cdots, n$ and

$$
\left(\sigma_{t, F} \circ_{h} \sigma_{v, R}\right)(\gamma)=R_{n}^{n}\left(F, \widehat{\sigma}_{t, F}\left[r_{1}\right], \cdots, \widehat{\sigma}_{t, F}\left[r_{m}\right]\right)=\gamma\left(\widetilde{s}_{1}, \cdots, \widetilde{s}_{n}\right)
$$

where $\widetilde{s}_{p}=S_{n}^{n}\left(s_{p}, \widehat{\sigma}_{t, F}\left[r_{1}\right], \cdots, \widehat{\sigma}_{t, F}\left[r_{m}\right]\right)$ for all $p=1, \cdots, n$.

Since $w_{c_{i}^{\prime}}=x_{c_{i}}$ and $u_{c_{i}}=x_{a_{i}}=x_{\pi_{1}^{-1}\left(c_{i}^{\prime}\right)}$ for all $i=1, \cdots, k$, we have $\widetilde{w}_{c_{i}^{\prime}}=S_{m}^{m}\left(w_{c_{i}^{\prime}}, \widehat{\sigma}_{w, H}\left[u_{1}\right], \cdots, \widehat{\sigma}_{w, H}\left[u_{m}\right]\right)=S_{m}^{m}\left(x_{c_{i}}, \widehat{\sigma}_{w, H}\left[u_{1}\right], \cdots, \widehat{\sigma}_{w, H}\left[u_{m}\right]\right)=\widehat{\sigma}_{w, H}\left[u_{c_{i}}\right]=$ $x_{\pi_{1}^{-1}\left(c_{i}^{\prime}\right)}$ for all $i=1, \cdots, k$. Similarly, $\widetilde{t}_{a_{i}^{\prime}}=x_{\pi_{2}^{-1}\left(a_{i}^{\prime}\right)}, \widetilde{h}_{d_{j}^{\prime}}=x_{\phi_{1}^{-1}\left(d_{j}^{\prime}\right)}$ and $\widetilde{s}_{b_{j}^{\prime}}=x_{\phi_{2}^{-1}\left(b_{j}^{\prime}\right)}$ for all $i=1, \cdots, k$ and $j=1, \cdots, l$. Next, we consider

$$
\begin{aligned}
& \left(\sigma_{\pi_{1}(t), \phi_{1}(F)}{ }^{\circ_{h}} \sigma_{w, H} \circ_{h} \sigma_{u, E}\right)(f)=\widehat{\sigma}_{\pi_{1}(t), \phi_{1}(F)}\left[\left(\sigma_{w, H} \circ_{h} \sigma_{u, E}\right)(f)\right] \\
& =\widehat{\sigma}_{\pi_{1}(t), \phi_{1}(F)}\left[f\left(\widetilde{w}_{1}, \cdots, \widetilde{w}_{m}\right)\right] \\
& =S_{m}^{m}\left(\pi_{1}(t), \widehat{\sigma}_{\pi_{1}(t), \phi_{1}(F)}\left[\widetilde{w}_{1}\right], \cdots, \widehat{\sigma}_{\pi_{1}(t), \phi_{1}(F)}\left[\widetilde{w}_{m}\right]\right) \\
& =\pi_{1}^{-1}\left(\pi_{1}(t)\right) \text {, } \\
& \text { since } I\left(\pi_{1}(t)\right)=\left\{c_{1}^{\prime}, \cdots, c_{k}^{\prime}\right\} \text { and } \\
& \widehat{\sigma}_{\pi_{1}(t), \phi_{1}(F)}\left[\widetilde{w}_{c_{i}^{\prime}}\right]=x_{\pi_{1}^{-1}\left(c_{i}^{\prime}\right)} \text { for all } i=1, \cdots, k, \\
& =t=\sigma_{t, F}(f)
\end{aligned}
$$

and $\left(\sigma_{\pi_{1}(t), \phi_{1}(F)}{ }^{\circ}{ }_{h} \sigma_{w, H} \sigma_{u, E}\right)(\gamma)=\phi_{1}^{-1}\left(\phi_{1}(F)\right)=F=\sigma_{t, F}(\gamma)$. So $\sigma_{\pi_{1}(t), \phi_{1}(F)}{ }^{\circ_{h}} \sigma_{w, H} \circ_{h}$ $\sigma_{u, E}=\sigma_{t, F}$. Similarly, we can show that $\sigma_{w, H}=\sigma_{\pi_{2}(w), \phi_{2}(H)} \circ_{h} \sigma_{t, F} \circ_{h} \sigma_{v, R}$. Therefore $\sigma_{t, F} \mathcal{J} \sigma_{w, H}$.

Theorem 3.10. In $R_{T}, \mathcal{J}=\mathcal{D}$.

Proof. It is obvious that $\mathcal{D} \subseteq \mathcal{J}$. We will show that $\mathcal{J} \subseteq \mathcal{D}$. Let $\sigma_{t, F}, \sigma_{w, H} \in R_{T}$ such that $\sigma_{t, F} \mathcal{J} \sigma_{w, H}$ where $t=f\left(t_{1}, \cdots, t_{m}\right), w=f\left(w_{1}, \cdots, w_{m}\right) \in W_{(m)}\left(X_{m}\right)$ and $F=$ $\gamma\left(s_{1}, \cdots, s_{n}\right), H=\gamma\left(h_{1}, \cdots, h_{n}\right) \in r F_{((m),(n))}\left(X_{n}\right)$. By Theorem 3.9, we obtain that $|I(w)|=$ $|I(t)|$ and $|I(H)|=|I(F)|$. Let $I(t)=\left\{a_{1}, \cdots, a_{k}\right\}, I(w)=\left\{c_{1}, \cdots, c_{k}\right\}, I(F)=\left\{b_{1}, \cdots, b_{l}\right\}$ and $I(H)=\left\{d_{1}, \cdots, d_{l}\right\}$. Since $\sigma_{t, F}, \sigma_{w, H} \in R_{T}$, there are subsets $\left\{a_{1}^{\prime}, \cdots, a_{k}^{\prime}\right\},\left\{c_{1}^{\prime}, \cdots, c_{k}^{\prime}\right\}$ of $\{1, \cdots, m\}$ and subsets $\left\{b_{1}^{\prime}, \cdots, b_{l}^{\prime}\right\},\left\{d_{1}^{\prime}, \cdots, d_{l}^{\prime}\right\}$ of $\{1, \cdots, n\}$ such that $t_{a_{i}^{\prime}}=x_{a_{i}}, w_{c_{i}^{\prime}}=$ $x_{c_{i}}, s_{b_{j}^{\prime}}=x_{b_{j}}$ and $h_{d_{l}^{\prime}}=x_{d_{j}}$ for all $i=1, \cdots, k$ and $j=1, \cdots, l$. We will show that $\sigma_{t, F} \mathcal{D} \sigma_{w, H}$, i.e. there is $\sigma_{p, G} \in \operatorname{Relhyp}((m),(n))$ such that $\sigma_{w, H} \mathcal{L} \sigma_{p, G}$ and $\sigma_{p, G} \mathcal{R} \sigma_{t, F}$. Since $|I(w)|=|I(t)|$ and $|I(H)|=|I(F)|$, we define bijective maps $\pi: I(t) \rightarrow I(w)$ by $\pi\left(a_{i}\right)=c_{i}$, for all $i=1, \cdots, k$, and $\phi: I(F) \rightarrow I(H)$ by $\pi\left(b_{j}\right)=d_{j}$, for all $j=1, \cdots, l$. Choose $\sigma_{p, G} \in$ $\operatorname{Relhyp}((m),(n))$ such that $p=\pi(t)$ and $G=\phi(F)$. Then $I(p)=\left\{\pi\left(a_{1}\right), \cdots, \pi\left(a_{k}\right)\right\}=$ $\left\{c_{1}, \cdots, c_{k}\right\}=I(w)$ and $I(G)=\left\{\phi\left(b_{1}\right), \cdots, \phi\left(b_{l}\right)\right\}=\left\{d_{1}, \cdots, d_{l}\right\}=I(H)$. By Theorem 3.7, we have $\sigma_{w, H} \mathcal{L} \sigma_{p, G}$. Next, we will show that $\sigma_{p, G} \mathcal{R} \sigma_{t, F}$, i.e. there are $\sigma_{u, E}, \sigma_{v, G} \in$ $\operatorname{Relhyp}((m),(n))$ such that $\sigma_{p, G}=\sigma_{t, F} \circ_{h} \sigma_{u, E}$ and $\sigma_{t, F}=\sigma_{p, G} \circ_{h} \sigma_{v, G}$. Choose $\sigma_{u, E}, \sigma_{v, G} \in$ 
$\operatorname{Relhyp}((m),(n))$ with $u=f\left(u_{1}, \cdots, u_{m}\right), v=f\left(v_{1}, \cdots, v_{m}\right) \in W_{(m)}\left(X_{m}\right)$ and $E=$ $\gamma\left(e_{1}, \cdots, e_{n}\right), G=\gamma\left(g_{1}, \cdots, g_{n}\right) \in r F_{((m),(n))}\left(X_{n}\right)$ such that $u_{a_{i}}=x_{c_{i}}, v_{c_{i}}=x_{a_{i}}, e_{b_{j}}=x_{d_{j}}$ and $g_{d_{j}}=x_{b_{j}}$ for all $i=1, \cdots, k$ and $j=1, \cdots, l$. By calculation, we obtain that $\sigma_{p, G} \mathcal{R} \sigma_{t, F}$. Therefore $\sigma_{t, F} \mathcal{D} \sigma_{w, H}$.

Theorem 3.11. Let $\sigma_{t, F}, \sigma_{w, H} \in R_{T}$. Then $\sigma_{t, F} \mathcal{H} \sigma_{w, H}$ if and only if

(i) $I(w)=I(t)$ and $I(H)=I(F)$,

(ii) $w=\pi(t)$ and $H=\phi(F)$ where $\pi$ and $\phi$ are bijective maps on $I(t)$ and $I(F)$, respectively.

Proof. The proof is completed by Theorem 3.7 and Theorem 3.8 .

\section{Acknowledgments}

The authors are highly grateful to the referees for their valuable comments and suggestions for improving the article. This research was supported by Chiang Mai University, Chiang Mai 50200, Thailand.

\section{References}

[1] T. Changphas and K. Denecke, All idempotent hypersubstitutions of type $(2,2)$, Semigroup Forum, 76 (2008), 525-539.

[2] T. Changphas and K. Denecke, The order of hypersubstitution of type (2,2), Acta Mathematica Sinica, 23 (2007), 659-670.

[3] J. Daengsaen and S. Leeratanavalee, Regularity of relational hypersubstitutions for algebraic systems, JP Journal of Algebra, Number Theory and Applications, 44 (2019), 229-250.

[4] K. Denecke and J. Koppitz, Finite monoids of hypersubstitutions of type (2), Semigroup Forum, 52 (1998), 265-275.

[5] K. Denecke, D. Lau, R. Pöschel and D. Schweigert, Hyperidentities, hyperequatinal classes and clone congruences, Contributions to General Algebra(Verlag Hölder-Pichler-Tempsky, Wien), 7 (1991), 97-118.

[6] K. Denecke and D. Phusanga, Hyperformulas and Solid Algebraic Systems, Studia Logica, 90 (2008), 263-286. 
[7] T. Kumduang and S. Leeratanavalee, Regularity of linear hypersubstitution for algebraic systems of type $((n),(m))$, Communications in Mathematics and Applications, 10 (2019), $1-18$.

[8] J. Koppitz and K. Denecke, M-Solid Varieties of Algebras, Springer Science and Business Media, New York, 2006.

[9] N. Lekkoksung and D. Phusanga, The order of Linear Relational Hypersubstitutions for Algebraic Systems, Special Issue The 14th IMT-GT ICMSA 2018, Thai Journal of Mathematics (2019), 83-91.

[10] A. I. Mal'cev, Algebraic Systems, Akademie-Verlag, Berlin, 1973.

[11] D. Phusanga, A Binary Relation on Sets of Hypersubstitutions for Algebraic Systems, Southeast Asian Bulletin of Mathematics, 44 (2020), 255-269.

[12] D. Phusanga, Some varieties of algebraic systems of type $((n),(m))$, Asian-European Journal of Mathematics, 12 (2019), 1950005.

[13] D. Phusanga and J. Koppitz, The monoid of hypersubstitutions for algebraic systems, Announcements of Union of Scientists Silven, 33 (2018), 119-129.

[14] W. Puninagool and S. Leeratanavalee, The monoid of generalized hypersubstitutions of type $\tau=(n)$, Discussiones Mathematicae : General Algebra and Applications, 30 (2010), $173-191$.

[15] Sh. L. Wismath, The monoid of hypersubstitutions of type $(n)$, Southest Asian Bulletin of Mathematics, 24 (2000), 115-128.

[16] W. Wongpinit and S. Leeratanavalee, All maximal idempotent submonoids of $H y p_{G}(n)$, Surveys in Mathematics and its Applications, 10 (2015), 41-48.

Jukkrit Daengsaen Department of Mathematics, Faculty of Science, Chiang Mai University, Chiang Mai 50200, Thailand

E-mail: daengsaen.j@gmail.com

Sorasak Leeratanavalee Research Center in Mathematics and Applied Mathematics, Department of Mathematics, Faculty of Science, Chiang Mai University, Chiang Mai 50200, Thailand E-mail: sorasak.1@cmu.ac.th 